Universidad

:\% de Alcalá

\title{
BIBLIOTECA
}

Document downloaded from the institutional repository of the University of Alcala: https://ebuah.uah.es/dspace/

This is a postprint version of the following published document:

Martín, A. et al., 2013. Copper(I) and silver(I) complexes supported by the tridentate $[\{\mathrm{Ti}(n(5)-\mathrm{C} 5 \mathrm{Me} 5)(\mu-\mathrm{NH})\} 3(\mu 3-\mathrm{N})]$ metalloligand. Inorganic chemistry, 52(2), pp.918-930.

Available at https://doi.org/10.1021/ic302113k

C 2012 American Chemical Society.

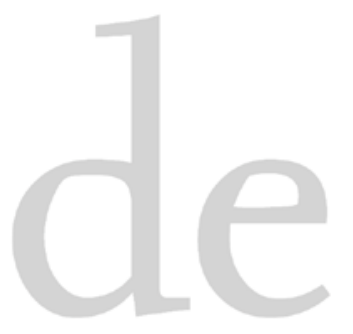

(Article begins on next page)

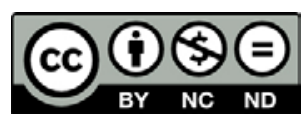

This work is licensed under a

Creative Commons Attribution-NonCommercial-NoDerivatives

4.0 International License. 


\section{Copper(I) and Silver(I) Complexes Supported by the Tridentate}

\section{$\left[\left\{\mathrm{Ti}\left(\eta^{5}-\mathrm{C}_{5} \mathrm{Me}_{5}\right)(\mu-\mathrm{NH})\right\}_{3}\left(\mu_{3}-\mathrm{N}\right)\right]$ Metalloligand}

Avelino Martín, Noelia Martínez-Espada, Miguel Mena, Adrián Pérez-Redondo, and Carlos Yélamos*

Departamento de Química Inorgánica, Universidad de Alcalá. 28871 Alcalá de HenaresMadrid (Spain). FAX: (+34) 91-8854683. E-mail: carlos.yelamos@uah.es

\section{Table of Contents Synopsis:}

Copper(I) and silver(I) complexes $\left[\left(\mathrm{CF}_{3} \mathrm{SO}_{2} \mathrm{O}\right) \mathrm{M}\left\{\left(\mu_{3}-\mathrm{NH}\right)_{3} \mathrm{Ti}_{3}\left(\eta^{5}-\mathrm{C}_{5} \mathrm{Me}_{5}\right)_{3}\left(\mu_{3}-\mathrm{N}\right)\right\}\right]$ react with a variety of donor molecules $\mathrm{L}$ to give the ionic compounds $\left[(\mathrm{L}) \mathrm{M}\left\{\left(\mu_{3}-\mathrm{NH}\right)_{3} \mathrm{Ti}_{3}\left(\eta^{5}-\right.\right.\right.$ $\left.\left.\left.\mathrm{C}_{5} \mathrm{Me}_{5}\right)_{3}\left(\mu_{3}-\mathrm{N}\right)\right\}\right]\left[\mathrm{O}_{3} \mathrm{SCF}_{3}\right]\left(\mathrm{L}=\mathrm{NH}_{3}, \mathrm{py}, \mathrm{CNAr}, \mathrm{CN} t \mathrm{Bu}, \mathrm{PPh}_{3}\right)$ whose cations contain $\left[\mathrm{MTi}_{3} \mathrm{~N}_{4}\right]$ cube-type cores. The analogous treatment with bisphosphanes $\mathrm{Ph}_{2} \mathrm{P}\left(\mathrm{CH}_{2}\right)_{n} \mathrm{PPh}_{2}$ $(\mathrm{n}=1,2)$ gives $1: 1$ adducts $\left[(\mathrm{dppm}) \mathrm{M}\left\{\left(\mu_{3}-\mathrm{NH}\right)_{3} \mathrm{Ti}_{3}\left(\eta^{5}-\mathrm{C}_{5} \mathrm{Me}_{5}\right)_{3}\left(\mu_{3}-\mathrm{N}\right)\right\}\right]\left[\mathrm{O}_{3} \mathrm{SCF}_{3}\right]$ or $2: 1$ systems where a dppm or dppe ligand bridge two cube-type cations.

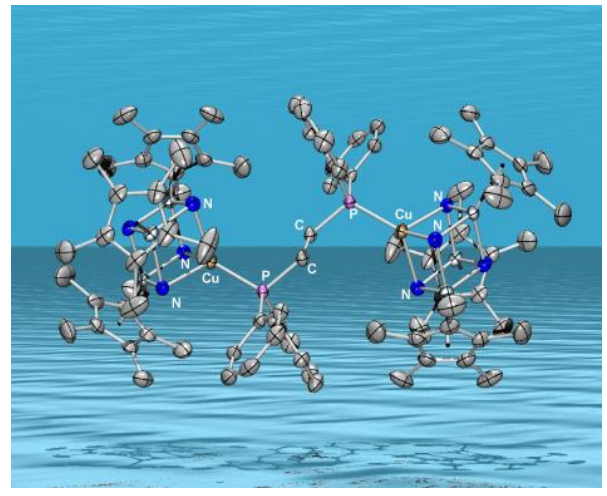




\section{Abstract:}

Copper(I) and silver(I) ionic compounds $\left[(\mathrm{L}) \mathrm{M}\left\{\left(\mu_{3}-\mathrm{NH}\right)_{3} \mathrm{Ti}_{3}\left(\eta^{5}-\mathrm{C}_{5} \mathrm{Me}_{5}\right)_{3}\left(\mu_{3}-\mathrm{N}\right)\right\}\right]\left[\mathrm{O}_{3} \mathrm{SCF}_{3}\right]$ containing $\left[\mathrm{MTi}_{3} \mathrm{~N}_{4}\right]$ cube-type cations have been prepared by reaction of $\left[\left(\mathrm{CF}_{3} \mathrm{SO}_{2} \mathrm{O}\right) \mathrm{M}\left\{\left(\mu_{3}-\mathrm{NH}\right)_{3} \mathrm{Ti}_{3}\left(\eta^{5}-\mathrm{C}_{5} \mathrm{Me}_{5}\right)_{3}\left(\mu_{3}-\mathrm{N}\right)\right\}\right](\mathrm{M}=\mathrm{Cu}(\mathbf{2}), \mathrm{Ag}(\mathbf{3}))$ with a variety of donor molecules L. Treatment of complexes 2 and $\mathbf{3}$ with $\mathrm{NH}_{3}$ in toluene at room temperature gives the ammonia adducts $\left[\left(\mathrm{H}_{3} \mathrm{~N}\right) \mathrm{M}\left\{\left(\mu_{3}-\mathrm{NH}\right)_{3} \mathrm{Ti}_{3}\left(\eta^{5}-\mathrm{C}_{5} \mathrm{Me}_{5}\right)_{3}\left(\mu_{3}-\right.\right.\right.$ $\mathrm{N})\}]\left[\mathrm{O}_{3} \mathrm{SCF}_{3}\right](\mathrm{M}=\mathrm{Cu}(\mathbf{4}), \mathrm{Ag}(\mathbf{5}))$, whose X-ray crystal structures reveal two cube-type cations associated through hydrogen bonding interactions between the ammine ligands and one oxygen atom of each trifluoromethanesulfonate anion. Analogous treatment of $\mathbf{2}$ and $\mathbf{3}$ with one equivalent of pyridine, 2,6-dimethylphenylisocyanide, tert-butylisocyanide, or triphenylphosphane gives the ionic compounds $\left[(\mathrm{L}) \mathrm{M}\left\{\left(\mu_{3}-\mathrm{NH}\right)_{3} \mathrm{Ti}_{3}\left(\eta^{5}-\mathrm{C}_{5} \mathrm{Me}_{5}\right)_{3}\left(\mu_{3^{-}}\right.\right.\right.$ N) $\}]\left[\mathrm{O}_{3} \mathrm{SCF}_{3}\right](\mathrm{L}=\mathrm{py}, \mathrm{M}=\mathrm{Cu}(6), \mathrm{Ag}(7) ; \mathrm{L}=\mathrm{CNAr}, \mathrm{M}=\mathrm{Cu}(\mathbf{8}), \mathrm{Ag}(\mathbf{9}) ; \mathrm{L}=\mathrm{CN} t \mathrm{Bu}, \mathrm{M}$ $\left.=\mathrm{Cu}(\mathbf{1 0}), \mathrm{Ag}(\mathbf{1 1}) ; \mathrm{L}=\mathrm{PPh}_{3}, \mathrm{M}=\mathrm{Cu}(\mathbf{1 2}), \mathrm{Ag}(\mathbf{1 3})\right)$. The reactions of 2 and 3 with methylenebis(diphenylphosphane) (dppm) in a 1:1 ratio lead to the single-cube complexes $\left[(\mathrm{dppm}) \mathrm{M}\left\{\left(\mu_{3}-\mathrm{NH}\right)_{3} \mathrm{Ti}_{3}\left(\eta^{5}-\mathrm{C}_{5} \mathrm{Me}_{5}\right)_{3}\left(\mu_{3}-\mathrm{N}\right)\right\}\right]\left[\mathrm{O}_{3} \mathrm{SCF}_{3}\right](\mathrm{M}=\mathrm{Cu}(\mathbf{1 4}), \mathrm{Ag}(\mathbf{1 5}))$, whereas in a 2:1 ratio give the bisphosphane-bridged double-cube compounds $\left[\left\{\mathrm{M}\left(\mu_{3}-\mathrm{NH}\right)_{3} \mathrm{Ti}_{3}\left(\eta^{5}-\right.\right.\right.$ $\left.\left.\left.\mathrm{C}_{5} \mathrm{Me}_{5}\right)_{3}\left(\mu_{3}-\mathrm{N}\right)\right\}_{2}(\mu-\mathrm{dppm})\right]\left[\mathrm{O}_{3} \mathrm{SCF}_{3}\right]_{2}(\mathrm{M}=\mathrm{Cu}(\mathbf{1 6}), \mathrm{Ag}(\mathbf{1 7}))$. Similarly, treatment of 2 or 3 with a half equivalent of ethane-1,2-diylbis(diphenylphosphane) (dppe) affords doublecube derivatives $\left[\left\{\mathrm{M}\left(\mu_{3}-\mathrm{NH}\right)_{3} \mathrm{Ti}_{3}\left(\eta^{5}-\mathrm{C}_{5} \mathrm{Me}_{5}\right)_{3}\left(\mu_{3}-\mathrm{N}\right)\right\}_{2}(\mu-d p p e)\right]\left[\mathrm{O}_{3} \mathrm{SCF}_{3}\right]_{2}(\mathrm{M}=\mathrm{Cu}(\mathbf{1 8})$, $\operatorname{Ag}(\mathbf{1 9}))$. The X-ray crystal structures of $4,5,10,14,15$, and 18 have been determined. 


\section{Introduction}

An extensive coordination chemistry of copper and silver has been developed with tridentate nitrogen-based ligands. Among the numerous ligands pursued in this vein, the family of the anionic tris(pyrazolyl)borates is the most widespread because of their ability to serve as versatile facially coordinating six-electron donors to metal ions. ${ }^{1}$ The electronic properties and especially the flexible coordination ability of these tripodal ligands have permitted the isolation of a large number of relevant novel species of copper(I) and silver(I). ${ }^{2,3}$ In comparison, the chemistry of tris(pyrazolyl)methane ligands, ${ }^{1 b, 4}$ the neutral analogues of the tris(pyrazolyl)borates, has been traditionally underdeveloped, although many copper and silver complexes with these ligands are known in nowadays. ${ }^{5}$ More rigid neutral fac-coordinating $\mathrm{N}_{3}$-donor ligands employed in group 11 metal complexes are cyclic triamines such as 1,4,7-triazacyclononanes ${ }^{6}$ and 1,3,5-triazacyclohexanes. ${ }^{7}$

As part of a program devoted to the development of polynuclear nitrido complexes, ${ }^{8}$ we have been studying the coordination chemistry of the trinuclear titanium imido-nitrido complex $\left[\left\{\mathrm{Ti}\left(\eta^{5}-\mathrm{C}_{5} \mathrm{Me}_{5}\right)(\mu-\mathrm{NH})\right\}_{3}\left(\mu_{3}-\mathrm{N}\right)\right]^{9,10}(\mathbf{1})$. Complex 1 shows a six-membered $\left[\mathrm{Ti}_{3}(\mu-\right.$ $\mathrm{NH})_{3}$ ] ring with three $\mathrm{NH}$ electron-donor imido groups and exhibits a strong resemblance to 1,4,7-triazacyclononane and, especially, 1,3,5-triazacyclohexane ligands. This similarity prompted us to explore its potential application as a tridentate, preorganized, ${ }^{11}$ metalloligand toward a variety of metals. Thus, our group has reported extensively about the use of $\mathbf{1}$ as a neutral tridentate chelate ligand among the transition, ${ }^{12}$ lanthanide, ${ }^{13}$ and main-group metals. ${ }^{14}$ In particular, we have demonstrated that complex $\mathbf{1}$ entraps one equivalent of copper(I) or silver(I) trifluoromethanesulfonate to give the adducts

$\left[\left(\mathrm{CF}_{3} \mathrm{SO}_{2} \mathrm{O}\right) \mathrm{M}\left\{\left(\mu_{3}-\mathrm{NH}\right)_{3} \mathrm{Ti}_{3}\left(\eta^{5}-\mathrm{C}_{5} \mathrm{Me}_{5}\right)_{3}\left(\mu_{3}-\mathrm{N}\right)\right\}\right] \quad\left(\mathrm{M}=\mathrm{Cu}(\mathbf{2}),{ }^{15} \mathrm{Ag}(\mathbf{3})^{16}\right)$. The poor 
solubility in hydrocarbon solvents and the IR spectroscopic data of compounds $\mathbf{2}$ and $\mathbf{3}$ suggested a weak interaction between the triflate anions and the group 11 metal ions. Given the poor coordinating capability of the trifluoromethanesulfonate anion, ${ }^{17}$ we became interested in the use of complexes $\mathbf{2}$ and $\mathbf{3}$ as precursors to a range of derivatives formed by substitution of this labile ligand by ligands of greater nucleophilicity. Herein we describe the results obtained in the treatment of $\mathbf{2}$ and $\mathbf{3}$ with a variety of donor molecules $\mathrm{L}$ to produce a series of ionic complexes $\left[(\mathrm{L}) \mathrm{M}\left\{\left(\mu_{3}-\mathrm{NH}\right)_{3} \mathrm{Ti}_{3}\left(\eta^{5}-\mathrm{C}_{5} \mathrm{Me}_{5}\right)_{3}\left(\mu_{3}-\mathrm{N}\right)\right\}\right]\left[\mathrm{O}_{3} \mathrm{SCF}_{3}\right]$. The synthesis, structures, and properties of the new compounds are discussed and compared with other copper and silver derivatives containing tridentate nitrogen-based ligands. 


\section{Experimental Section}

General Considerations. All manipulations were carried out under argon atmosphere using Schlenk line or glovebox techniques. Hexane and toluene were distilled from $\mathrm{Na} / \mathrm{K}$ alloy just before use. Dichloromethane was dried with $\mathrm{P}_{2} \mathrm{O}_{5}$ and distilled prior to use. Fluorobenzene was dried with $\mathrm{CaH}_{2}$ and distilled prior to use. NMR solvents were dried with $\mathrm{Na} / \mathrm{K}$ alloy $\left(\mathrm{C}_{6} \mathrm{D}_{6}\right)$ or calcium hydride $\left(\mathrm{CDCl}_{3}, \mathrm{CD}_{2} \mathrm{Cl}_{2}\right)$ and vacuum-distilled. Ovendried glassware was repeatedly evacuated with a pumping system (ca. $1 \times 10^{-3}$ Torr) and subsequently filled with inert gas. The syntheses and characterization of silver derivatives were carried in the dark by using amber stained glassware in all the manipulations. Electronic grade ammonia (purity $>99.995 \%, \mathrm{O}_{2}<2 \mathrm{ppm}$, and $\mathrm{H}_{2} \mathrm{O}<10 \mathrm{ppm}$ ) was purchased from Sociedad Española del Oxígeno, and used as received. Pyridine (purchased from Aldrich) was distilled from $\mathrm{CaH}_{2}$. 2,6-Dimethylphenylisocyanide, tertbutylisocyanide, methylenebis(diphenylphosphane) (dppm) and ethane-1,2diylbis(diphenylphosphane) (dppe) were purchased from Aldrich and used as received. Triphenylphosphane was purchased from Aldrich and sublimed under vacuum prior to use. $\left[\left(\mathrm{CF}_{3} \mathrm{SO}_{2} \mathrm{O}\right) \mathrm{Cu}\left\{\left(\mu_{3}-\mathrm{NH}\right)_{3} \mathrm{Ti}_{3}\left(\eta^{5}-\mathrm{C}_{5} \mathrm{Me}_{5}\right)_{3}\left(\mu_{3}-\mathrm{N}\right)\right\}\right] \cdot \mathrm{C}_{7} \mathrm{H}_{8}\left(2 \cdot \mathrm{C}_{7} \mathrm{H}_{8}\right)^{15}$ and $\left[\left(\mathrm{CF}_{3} \mathrm{SO}_{2} \mathrm{O}\right) \mathrm{Ag}\left\{\left(\mu_{3}-\right.\right.\right.$ $\left.\left.\mathrm{NH})_{3} \mathrm{Ti}_{3}\left(\eta^{5}-\mathrm{C}_{5} \mathrm{Me}_{5}\right)_{3}\left(\mu_{3}-\mathrm{N}\right)\right\}\right](\mathbf{3})^{16}$ were prepared according to published procedures.

Samples for infrared spectroscopy were prepared as $\mathrm{KBr}$ pellets and the spectra were obtained using an FT-IR Perkin Elmer SPECTRUM 2000 spectrophotometer. ${ }^{1} \mathrm{H},{ }^{13} \mathrm{C}\left\{{ }^{1} \mathrm{H}\right\}$, ${ }^{19} \mathrm{~F}$ and ${ }^{31} \mathrm{P}\left\{{ }^{1} \mathrm{H}\right\}$ NMR spectra were recorded on a Varian Unity-300 and/or Unity-500 Plus spectrometers. Chemical shifts $\left(\delta\right.$, ppm) in the ${ }^{1} \mathrm{H}$ and ${ }^{13} \mathrm{C}\left\{{ }^{1} \mathrm{H}\right\}$ NMR spectra are given relative to residual protons or to carbon of the solvent. Chemical shifts $(\delta, \mathrm{ppm})$ in the ${ }^{19} \mathrm{~F}$ 
and ${ }^{31} \mathrm{P}\left\{{ }^{1} \mathrm{H}\right\}$ NMR spectra are given relative to $\mathrm{CFCl}_{3}$ or $\mathrm{H}_{3} \mathrm{PO}_{4}$ as external references. Microanalyses (C, H, N, S) were performed in a Leco CHNS-932 microanalyzer. Synthesis of $\left[\left(\mathbf{H}_{3} \mathbf{N}\right) \mathrm{Cu}\left\{\left(\mu_{3}-\mathbf{N H}\right)_{3} \mathbf{T i}_{3}\left(\eta^{5}-\mathbf{C}_{5} \mathbf{M e}_{5}\right)_{3}\left(\mu_{3}-\mathbf{N}\right)\right\}\right]\left[\mathbf{O}_{3} \mathrm{SCF}_{3}\right]$ (4). A $100 \mathrm{~mL}$ ampule (Teflon stopcock) was charged with $2 \cdot \mathrm{C}_{7} \mathrm{H}_{8}(0.15 \mathrm{~g}, 0.16 \mathrm{mmol})$ and toluene $(20 \mathrm{~mL})$. After cooling at $-78{ }^{\circ} \mathrm{C}$, the argon atmosphere was changed by $\mathrm{NH}_{3}$. The reaction mixture was allowed to warm to room temperature and stirred for $16 \mathrm{~h}$. The volatile components were removed under reduced pressure and the resultant orange solid was washed with toluene (5 $\mathrm{mL})$ and vacuum-dried to give $4(0.10 \mathrm{~g}, 77 \%) . \mathrm{IR}\left(\mathrm{KBr}, \mathrm{cm}^{-1}\right): \tilde{v} 3383(\mathrm{w}), 3355(\mathrm{w}), 3346$ (m), 3306 (s), 3266 (w), 3223 (w), 3184 (w), 2910 (s), 2859 (m), 1620 (m), 1490 (w), 1429 (m), 1378 (s), 1266 (vs), 1223 (s), 1156 (vs), 1031 (vs), 785 (m), 729 (s), 695 (m), 646 (vs), $572(\mathrm{w}), 517(\mathrm{~m}), 476(\mathrm{w}), 433(\mathrm{~m}), 406(\mathrm{~m}) .{ }^{1} \mathrm{H}$ NMR $\left(300 \mathrm{MHz}, \mathrm{CDCl}_{3}, 20{ }^{\circ} \mathrm{C}\right): \delta 11.71$ (s broad, 3H; NH), 2.12 (s broad, 3H; $\left.\mathrm{NH}_{3}\right), 2.02\left(\mathrm{~s}, 45 \mathrm{H} ; \mathrm{C}_{5} \mathrm{Me}_{5}\right) .{ }^{13} \mathrm{C}\left\{{ }^{1} \mathrm{H}\right\}$ NMR (75 $\left.\mathrm{MHz}, \mathrm{CDCl}_{3}, 20^{\circ} \mathrm{C}\right): \delta 120.2\left(\mathrm{C}_{5} \mathrm{Me}_{5}\right), 11.6\left(\mathrm{C}_{5} \mathrm{Me}_{5}\right)$, the $\mathrm{CF}_{3}$ carbon atom resonance was not detected. ${ }^{19} \mathrm{~F}$ NMR $\left(282 \mathrm{MHz}, \mathrm{CDCl}_{3}, 20{ }^{\circ} \mathrm{C}\right): \delta-78.1\left(\mathrm{CF}_{3}\right)$. Anal. Calcd for $\mathrm{C}_{31} \mathrm{H}_{51} \mathrm{CuF}_{3} \mathrm{~N}_{5} \mathrm{O}_{3} \mathrm{STi}_{3}\left(M_{w}=837.98\right)$ : C 44.43, H 6.13, N 8.36, S 3.83. Found: $\mathrm{C} 44.13, \mathrm{H}$ 5.77, N 7.92, S 3.42 .

Synthesis of $\left[\left(\mathrm{H}_{3} \mathrm{~N}\right) \mathrm{Ag}\left\{\left(\mu_{3}-\mathrm{NH}\right)_{3} \mathrm{Ti}_{3}\left(\eta^{5}-\mathrm{C}_{5} \mathrm{Me}_{5}\right)_{3}\left(\mu_{3}-\mathrm{N}\right)\right\}\right]\left[\mathrm{O}_{3} \mathrm{SCF}_{3}\right]$ (5). In a fashion similar to the preparation of $\mathbf{4}$ but using an amber stained ampule, the reaction of $\mathbf{3}(0.15 \mathrm{~g}$, $0.17 \mathrm{mmol})$ in toluene $(20 \mathrm{~mL})$ with $\mathrm{NH}_{3}$ afforded $\mathbf{5} \cdot 0.5 \mathrm{C}_{7} \mathrm{H}_{8}$ as an orange solid $(0.080 \mathrm{~g}$, 50\%). IR (KBr, cm $\left.{ }^{-1}\right): \tilde{v} 3376(\mathrm{~m}), 3340(\mathrm{~m}), 3325(\mathrm{~m}), 3275(\mathrm{~m}), 3220(\mathrm{w}), 3183(\mathrm{w})$, 2947 (m), 2910 (s), 2859 (m), 1617 (w), 1604 (w), 1496 (w), 1452 (m), 1430 (m), 1380 (s), 1278 (vs), 1256 (vs), 1222 (s), 1153 (vs), 1030 (vs), 766 (m), 742 (s), 653 (vs), 638 (vs), $571(\mathrm{~m}), 517(\mathrm{~m}), 477(\mathrm{~m}), 428(\mathrm{~m}), 403(\mathrm{~m}) .{ }^{1} \mathrm{H}$ NMR $\left(300 \mathrm{MHz}, \mathrm{CDCl}_{3}, 20{ }^{\circ} \mathrm{C}\right): \delta 12.33$ 
(s broad, 3H; NH), 2.05 (s, 45H; $\mathrm{C}_{5} \mathrm{Me}_{5}$ ), 1.94 (s broad, 3H; $\left.\mathrm{NH}_{3}\right) .{ }^{13} \mathrm{C}\left\{{ }^{1} \mathrm{H}\right\}$ NMR $(75$ $\left.\mathrm{MHz}, \mathrm{CDCl}_{3}, 20^{\circ} \mathrm{C}\right): \delta 120.8\left(C_{5} \mathrm{Me}_{5}\right), 11.8\left(\mathrm{C}_{5} M e_{5}\right)$, the $\mathrm{CF}_{3}$ carbon atom resonance was not detected. ${ }^{19} \mathrm{~F}$ NMR $\left(282 \mathrm{MHz}, \mathrm{CDCl}_{3}, 20{ }^{\circ} \mathrm{C}\right): \delta-78.1\left(\mathrm{CF}_{3}\right)$. Anal. Calcd for $\mathrm{C}_{34.5} \mathrm{H}_{55} \mathrm{AgF}_{3} \mathrm{~N}_{5} \mathrm{O}_{3} \mathrm{STi}_{3}\left(M_{w}=928.38\right): \mathrm{C} 44.64, \mathrm{H}$ 5.97, N 7.54, S 3.45. Found: C 44.65, H 5.86, N 7.28, S 3.34.

Synthesis of $\left[(\mathbf{p y}) \mathbf{C u}\left\{\left(\mu_{3}-\mathbf{N H}\right)_{3} \mathbf{T i}_{3}\left(\eta^{5}-\mathrm{C}_{5} \mathbf{M e}_{5}\right)_{3}\left(\mu_{3}-\mathrm{N}\right)\right\}\right]\left[\mathbf{O}_{3} \mathbf{S C F}_{3}\right](6)$. A $100 \mathrm{~mL}$ Schlenk flask was charged with $\mathbf{2} \cdot \mathrm{C}_{7} \mathrm{H}_{8}(0.20 \mathrm{~g}, 0.22 \mathrm{mmol})$, pyridine $(0.020 \mathrm{~g}, 0.25 \mathrm{mmol})$ and toluene $(20 \mathrm{~mL})$. The reaction mixture was stirred at room temperature for $16 \mathrm{~h}$ to give an orange suspension. The volatile components were removed under reduced pressure, and the resultant solid was washed with toluene $(5 \mathrm{~mL})$ and vacuum-dried to afford $\mathbf{6} \cdot 0.5 \mathrm{C}_{7} \mathrm{H}_{8}$ as an orange solid $(0.17 \mathrm{~g}, 81 \%)$. IR $\left(\mathrm{KBr}, \mathrm{cm}^{-1}\right)$ : $\tilde{v} 3348$ (w), 3308 (m), 3024 (w), 2910 (s), $2859(\mathrm{~m}), 1603$ (m), 1495 (w), 1487 (w), 1445 (s), 1378 (s), 1276 (vs), 1259 (vs), 1223 (s), 1152 (vs), 1066 (w), 1031 (vs), 753 (s), 733 (s), 699 (m), 665 (s), 638 (vs), 571 (w), 517 (m), $476(\mathrm{~m}), 431(\mathrm{~m}), 404(\mathrm{~m}) .{ }^{1} \mathrm{H}$ NMR (300 MHz, $\left.\mathrm{CDCl}_{3}, 20^{\circ} \mathrm{C}\right): \delta 11.78$ (s broad, 3H; NH), 8.07 (m broad, 2H;o-py), 7.78 (m, 1H; p-py), 7.44 (m, 2H; m-py), 2.05 (s, 45H; $\left.\mathrm{C}_{5} \mathrm{Me}_{5}\right) \cdot{ }^{13} \mathrm{C}\left\{{ }^{1} \mathrm{H}\right\}$ NMR $\left(75 \mathrm{MHz}, \mathrm{CDCl}_{3}, 20{ }^{\circ} \mathrm{C}\right): \delta 150.9,138.3,125.8$ (py), 120.6 $\left(C_{5} \mathrm{Me}_{5}\right), 11.7\left(\mathrm{C}_{5} \mathrm{Me}_{5}\right)$, the $\mathrm{CF}_{3}$ carbon atom resonance was not detected. ${ }^{19} \mathrm{~F}$ NMR (282 $\left.\mathrm{MHz}, \mathrm{CDCl}_{3}, 20{ }^{\circ} \mathrm{C}\right): \delta-78.0\left(\mathrm{CF}_{3}\right)$. Anal. Calcd for $\mathrm{C}_{39.5} \mathrm{H}_{57} \mathrm{CuF}_{3} \mathrm{~N}_{5} \mathrm{O}_{3} \mathrm{STi}_{3}\left(M_{w}=946.12\right)$ : C 50.14, H 6.07, N 7.40, S 3.39. Found: C 50.19, H 5.84, N 6.99, S 2.87.

Synthesis of $\left[(\mathrm{py}) \mathrm{Ag}\left\{\left(\mu_{3}-\mathrm{NH}\right)_{3} \mathrm{Ti}_{3}\left(\eta^{5}-\mathrm{C}_{5} \mathrm{Me}_{5}\right)_{3}\left(\mu_{3}-\mathrm{N}\right)\right\}\right]\left[\mathrm{O}_{3} \mathrm{SCF}_{3}\right]$ (7). In a fashion similar to the preparation of $\mathbf{6}$, the treatment in an amber stained Schlenk flask of $\mathbf{3}(0.20 \mathrm{~g}, 0.23$ mmol) with pyridine $(0.020 \mathrm{~g}, 0.25 \mathrm{mmol})$ in toluene $(20 \mathrm{~mL})$ afforded $\mathbf{7} \cdot 0.5 \mathrm{C}_{7} \mathrm{H}_{8}$ as a green solid (0.18 g, 78\%). IR (KBr, cm $\left.{ }^{-1}\right): \tilde{v} 3358(\mathrm{w}), 3313(\mathrm{~m}), 3026(\mathrm{w}), 2911(\mathrm{~s}), 2859$ 
(m), 1602 (m), 1495 (m), 1445 (s), 1379 (s), 1279 (vs), 1261 (vs), 1222 (s), 1148 (vs), 1051 (m), 1031 (vs), 786 (s), 733 (s), 702 (s), 654 (vs), 637 (vs), 571 (m), 517 (m), 477 (m), 428 (m), $402(\mathrm{~m}) .{ }^{1} \mathrm{H}$ NMR (300 MHz, $\left.\mathrm{CDCl}_{3}, 20{ }^{\circ} \mathrm{C}\right): \delta 12.32$ (s broad, 3H; NH), 8.31 (m broad, 2H; o-py), 7.74 (m, 1H; p-py), 7.37 (m, 2H; m-py), 2.05 (s, 45H; $\left.\mathrm{C}_{5} \mathrm{Me}_{5}\right) .{ }^{13} \mathrm{C}\left\{{ }^{1} \mathrm{H}\right\}$ NMR (75 MHz, $\left.\mathrm{CDCl}_{3}, 20{ }^{\circ} \mathrm{C}\right): \delta 151.3,137.6,124.9$ (py), $120.7\left(C_{5} \mathrm{Me}_{5}\right), 11.8\left(\mathrm{C}_{5} M e_{5}\right)$, the $\mathrm{CF}_{3}$ carbon atom resonance was not detected. ${ }^{19} \mathrm{~F}$ NMR $\left(282 \mathrm{MHz}, \mathrm{CDCl}_{3}, 20{ }^{\circ} \mathrm{C}\right): \delta$ -77.8 $\left(\mathrm{CF}_{3}\right)$. Anal. Calcd for $\mathrm{C}_{39.5} \mathrm{H}_{57} \mathrm{AgF}_{3} \mathrm{~N}_{5} \mathrm{O}_{3} \mathrm{STi}_{3}\left(M_{w}=990.45\right): \mathrm{C} 47.90, \mathrm{H} 5.80, \mathrm{~N}$ 7.07, S 3.24. Found: C 47.88, H 5.69, N 6.59, S 3.25.

Synthesis of $\left[(\mathrm{ArNC}) \mathrm{Cu}\left\{\left(\mu_{3}-\mathrm{NH}\right)_{3} \mathrm{Ti}_{3}\left(\eta^{5}-\mathrm{C}_{5} \mathrm{Me}_{5}\right)_{3}\left(\mu_{3}-\mathrm{N}\right)\right\}\right]\left[\mathrm{O}_{3} \mathrm{SCF}_{3}\right]$ (8). In a fashion similar to the preparation of $\mathbf{6}$, the treatment of $2 \cdot \mathrm{C}_{7} \mathrm{H}_{8}(0.15 \mathrm{~g}, 0.16 \mathrm{mmol})$ with $2,6-$ dimethylphenylisocyanide $(0.022 \mathrm{~g}, 0.17 \mathrm{mmol})$ in toluene $(20 \mathrm{~mL})$ afforded $8 \cdot 0.5 \mathrm{C}_{7} \mathrm{H}_{8}$ as a green solid $(0.14 \mathrm{~g}, 88 \%)$. IR $\left(\mathrm{KBr}, \mathrm{cm}^{-1}\right)$ : $\tilde{v} 3364(\mathrm{~m}), 3282(\mathrm{~m}), 2912$ (s), 2859 (m), $2160(\mathrm{vs}, \mathrm{v}(\mathrm{CN})), 1603(\mathrm{w}), 1495(\mathrm{~m}), 1448(\mathrm{~m}), 1428(\mathrm{~m}), 1379(\mathrm{~m}), 1260$ (vs), $1222(\mathrm{~m})$, 1149 (s), 1031 (vs), 783 (m), 736 (m), $711(\mathrm{~m}), 648$ (vs), 636 (vs), $571(\mathrm{w}), 516(\mathrm{w}), 506$ (w), 479 (w), 437 (m), 407 (m). ${ }^{1} \mathrm{H}$ NMR (300 MHz, $\left.\mathrm{CDCl}_{3}, 20{ }^{\circ} \mathrm{C}\right): \delta 11.74$ (s broad, $3 \mathrm{H}$; $\mathrm{NH})$, 7.27-7.08 (m, 3H; $\left.\mathrm{C}_{6} \mathrm{H}_{3} \mathrm{Me}_{2}\right), 2.27$ (s, 6H; $\left.\mathrm{C}_{6} \mathrm{H}_{3} M e_{2}\right), 2.07$ (s, 45H; $\left.\mathrm{C}_{5} \mathrm{Me}_{5}\right) .{ }^{13} \mathrm{C}\left\{{ }^{1} \mathrm{H}\right\}$ NMR (75 MHz, $\mathrm{CDCl}_{3}, 20{ }^{\circ} \mathrm{C}$ ): $\delta 148.2$ (s broad, CN), 135.2, 130.1, 128.2, 125.5 $\left(C_{6} \mathrm{H}_{3} \mathrm{Me}_{2}\right), 121.3\left(C_{5} \mathrm{Me}_{5}\right), 121.0\left(\mathrm{q},{ }^{1} J(\mathrm{C}, \mathrm{F})=320 \mathrm{~Hz} ; \mathrm{CF}_{3}\right), 18.7\left(\mathrm{C}_{6} \mathrm{H}_{3} M e_{2}\right) 11.8$ $\left(\mathrm{C}_{5} \mathrm{Me}_{5}\right) .{ }^{19} \mathrm{~F}$ NMR $\left(282 \mathrm{MHz}, \mathrm{CDCl}_{3}, 20{ }^{\circ} \mathrm{C}\right): \delta-77.9 \quad\left(\mathrm{CF}_{3}\right)$. Anal. Calcd for $\mathrm{C}_{43.5} \mathrm{H}_{61} \mathrm{CuF}_{3} \mathrm{~N}_{5} \mathrm{O}_{3} \mathrm{STi}_{3}\left(M_{w}=998.20\right)$ : C 52.34, H 6.16, N 7.02, S 3.21. Found: C 52.26, H 6.27, N 6.26, S 2.94.

Synthesis of $\left[(\operatorname{ArNC}) \operatorname{Ag}\left\{\left(\mu_{3}-\mathrm{NH}\right)_{3} \mathrm{Ti}_{3}\left(\eta^{5}-\mathrm{C}_{5} \mathrm{Me}_{5}\right)_{3}\left(\mu_{3}-\mathrm{N}\right)\right\}\right]\left[\mathrm{O}_{3} \mathrm{SCF}_{3}\right]$ (9). In a fashion similar to the preparation of 7 , the treatment of $3(0.25 \mathrm{~g}, 0.29 \mathrm{mmol})$ with $2,6-$ 
dimethylphenylisocyanide $(0.040 \mathrm{~g}, 0.30 \mathrm{mmol})$ in toluene $(20 \mathrm{~mL})$ afforded $9 \cdot 0.5 \mathrm{C}_{7} \mathrm{H}_{8}$ as a green solid $(0.21 \mathrm{~g}, 70 \%)$. IR $\left(\mathrm{KBr}, \mathrm{cm}^{-1}\right): \tilde{v} 3358(\mathrm{w}), 3291$ (m), $2911(\mathrm{~s}), 2859(\mathrm{~m})$, 2177 (s, v(CN)), 1603 (w), 1490 (w), 1428 (m), 1379 (s), 1275 (vs), 1261 (vs), 1222 (s), 1146 (vs), 1031 (vs), 783 (s), 733 (s), 654 (vs), 636 (vs), 571 (w), 516 (m), 478 (m), 430 (m), 403 (m). ${ }^{1} \mathrm{H}$ NMR (300 MHz, $\left.\mathrm{CDCl}_{3}, 20{ }^{\circ} \mathrm{C}\right): \delta 12.31$ (s broad, 3H; NH), 7.29-7.11 (m, 3H; $\left.\mathrm{C}_{6} \mathrm{H}_{3} \mathrm{Me}_{2}\right), 2.32$ (s, 6H; $\left.\mathrm{C}_{6} \mathrm{H}_{3} M e_{2}\right), 2.07$ (s, 45H; $\left.\mathrm{C}_{5} \mathrm{Me}_{5}\right) .{ }^{13} \mathrm{C}\left\{{ }^{1} \mathrm{H}\right\}$ NMR (125 $\left.\mathrm{MHz}, \mathrm{CDCl}_{3}, 2{ }^{\circ} \mathrm{C}\right): \delta 148.9$ (s broad, $\left.\mathrm{CN}\right), 135.7,130.8,128.4,124.8\left(C_{6} \mathrm{H}_{3} \mathrm{Me}_{2}\right), 121.2$ $\left(C_{5} \mathrm{Me}_{5}\right), 121.0\left(\mathrm{q},{ }^{1} \mathrm{~J}(\mathrm{C}, \mathrm{F})=320 \mathrm{~Hz} ; \mathrm{CF}_{3}\right), 18.7\left(\mathrm{C}_{6} \mathrm{H}_{3} M e_{2}\right) 11.9\left(\mathrm{C}_{5} M e_{5}\right) .{ }^{19} \mathrm{~F}$ NMR $(282$ $\left.\mathrm{MHz}, \mathrm{CDCl}_{3}, 20{ }^{\circ} \mathrm{C}\right): \delta-77.9\left(\mathrm{CF}_{3}\right)$. Anal. Calcd for $\mathrm{C}_{43.5} \mathrm{H}_{61} \mathrm{AgF}_{3} \mathrm{~N}_{5} \mathrm{O}_{3} \mathrm{STi}_{3} \quad\left(M_{w}=\right.$ 1042.52): C 50.12, H 5.90, N 6.72, S 3.08. Found: C 49.44, H 5.58, N 6.46, S 2.64.

Synthesis of $\left[(t \mathrm{BuNC}) \mathrm{Cu}\left\{\left(\mu_{3}-\mathrm{NH}\right)_{3} \mathrm{Ti}_{3}\left(\eta^{5}-\mathrm{C}_{5} \mathrm{Me}_{5}\right)_{3}\left(\mu_{3}-\mathrm{N}\right)\right\}\right]\left[\mathrm{CF}_{3} \mathrm{SO}_{3}\right](10)$. In a fashion similar to the preparation of $\mathbf{6}$, the treatment of $\mathbf{2} \cdot \mathrm{C}_{7} \mathrm{H}_{8}(0.30 \mathrm{~g}, 0.33 \mathrm{mmol})$ with tertbutylisocyanide $(0.032 \mathrm{~g}, 0.38 \mathrm{mmol})$ in toluene $(20 \mathrm{~mL})$ afforded $\mathbf{1 0} \cdot 0.5 \mathrm{C}_{7} \mathrm{H}_{8}$ as a green solid (0.28 g, 90\%). IR (KBr, cm $\left.{ }^{-1}\right): \tilde{v} 3362(\mathrm{~m}), 3288(\mathrm{~m}), 2911(\mathrm{~s}), 2860(\mathrm{~m}), 2185$ (vs, $v(\mathrm{CN})), 1603$ (w), 1490 (m), 1452 (m), 1428 (m), 1379 (m), 1277 (vs), 1260 (vs), 1222 (m), 1147 (s), 1031 (s), 752 (m), 739 (m), 669 (m), 648 (vs), 637 (vs), 571 (w), 517 (m), 479 (w), 434 (m), 407 (m). ${ }^{1} \mathrm{H}$ NMR (300 MHz, $\left.\mathrm{CDCl}_{3}, 20{ }^{\circ} \mathrm{C}\right): \delta 11.69$ (s broad, 3H; NH), 2.04 (s, 45H; $\left.\mathrm{C}_{5} \mathrm{Me}_{5}\right), 1.42\left(\mathrm{~s}, 9 \mathrm{H} ; \mathrm{CMe}_{3}\right) .{ }^{13} \mathrm{C}\left\{{ }^{1} \mathrm{H}\right\} \mathrm{NMR}\left(75 \mathrm{MHz}, \mathrm{CDCl}_{3}, 20{ }^{\circ} \mathrm{C}\right): \delta 135.0$ (s broad, CN), $121.1\left(\mathrm{q},{ }^{1} J(\mathrm{C}, \mathrm{F})=320 \mathrm{~Hz} ; \mathrm{CF}_{3}\right), 121.0\left(C_{5} \mathrm{Me}_{5}\right), \quad 57.5\left(C \mathrm{Me}_{3}\right), 30.1$ $\left(\mathrm{CMe}_{3}\right), 11.7\left(\mathrm{C}_{5} \mathrm{Me}_{5}\right) .{ }^{19} \mathrm{~F} \mathrm{NMR}\left(282 \mathrm{MHz}, \mathrm{CDCl}_{3}, 20{ }^{\circ} \mathrm{C}\right): \delta-77.9\left(\mathrm{CF}_{3}\right)$. Anal. Calcd for $\mathrm{C}_{39.5} \mathrm{H}_{61} \mathrm{CuF}_{3} \mathrm{~N}_{5} \mathrm{O}_{3} \mathrm{STi}_{3}\left(M_{w}=950.15\right): \mathrm{C} 49.93, \mathrm{H}$ 6.47, N 7.37, S 3.37. Found: C 49.52, H 6.61, N 7.34, S 3.05. 
Synthesis of $\left[(t \mathrm{BuNC}) \mathrm{Ag}\left\{\left(\mu_{3}-\mathrm{NH}\right)_{3} \mathrm{Ti}_{3}\left(\eta^{5}-\mathrm{C}_{5} \mathrm{Me}\right)_{3}\left(\mu_{3}-\mathrm{N}\right)\right\}\right]\left[\mathrm{CF}_{3} \mathrm{SO}_{3}\right]$ (11). In a fashion similar to the preparation of 7 , the treatment of $3(0.30 \mathrm{~g}, 0.35 \mathrm{mmol})$ with tertbutylisocyanide $(0.032 \mathrm{~g}, 0.38 \mathrm{mmol})$ in toluene $(20 \mathrm{~mL})$ afforded $\mathbf{1 1} \cdot 0.5 \mathrm{C}_{7} \mathrm{H}_{8}$ as a green solid (0.26 g, 74\%). IR (KBr, cm $\left.{ }^{-1}\right): \tilde{v} 3360(\mathrm{~m}), 3295$ (m), 2913 (s), 2859 (m), 2202 (s, $v(\mathrm{CN})), 1603(\mathrm{w}), 1490(\mathrm{~m}), 1453(\mathrm{~m}), 1429(\mathrm{~m}), 1379(\mathrm{~s}), 1276(\mathrm{vs}), 1262(\mathrm{vs}), 1222(\mathrm{~m})$, $1200(\mathrm{~m}), 1149$ (vs), 1032 (vs), 880 (w), 791 (m), 752 (m), 731 (m), 655 (vs), 637 (vs), 571 (w), 517 (m), 478 (w), 430 (m), 404 (m). ${ }^{1} \mathrm{H}$ NMR (300 MHz, $\left.\mathrm{CDCl}_{3}, 20{ }^{\circ} \mathrm{C}\right): \delta 12.25$ (s broad, 3H; NH), 2.04 (s, 45H; $\left.\mathrm{C}_{5} \mathrm{Me}_{5}\right), 1.46\left(\mathrm{~s}, 9 \mathrm{H} ; \mathrm{CMe}_{3}\right) .{ }^{13} \mathrm{C}\left\{{ }^{1} \mathrm{H}\right\} \mathrm{NMR}(75 \mathrm{MHz}$, $\left.\mathrm{CDCl}_{3}, 20{ }^{\circ} \mathrm{C}\right): \delta 139.9(\mathrm{CN}), 121.1\left(\mathrm{C}_{5} \mathrm{Me}_{5}\right), 58.3\left(\mathrm{CMe}_{3}\right), 30.0\left(\mathrm{CMe}_{3}\right), 11.8\left(\mathrm{C}_{5} \mathrm{Me}_{5}\right)$, the $\mathrm{CF}_{3}$ carbon atom resonance was not detected. ${ }^{19} \mathrm{~F}$ NMR $\left(282 \mathrm{MHz}, \mathrm{CDCl}_{3}, 20{ }^{\circ} \mathrm{C}\right): \delta-77.9$ $\left(\mathrm{CF}_{3}\right)$. Anal. Calcd for $\mathrm{C}_{39.5} \mathrm{H}_{61} \mathrm{AgF}_{3} \mathrm{~N}_{5} \mathrm{O}_{3} \mathrm{STi}_{3}\left(M_{w}=994.48\right)$ : C 47.71, H 6.18, N 7.04, S 3.22. Found: C 46.94, H 6.34, N 6.84, S 2.97.

Synthesis of $\left[\left(\mathrm{Ph}_{3} \mathrm{P}\right) \mathrm{Cu}\left\{\left(\mu_{3}-\mathrm{NH}\right)_{3} \mathrm{Ti}_{3}\left(\eta^{5}-\mathrm{C}_{5} \mathrm{Me} 5\right)_{3}\left(\mu_{3}-\mathrm{N}\right)\right\}\right]\left[\mathrm{O}_{3} \mathrm{SCF}_{3}\right]$ (12). In a fashion similar to the preparation of $\mathbf{6}$, the treatment of $2 \cdot \mathrm{C}_{7} \mathrm{H}_{8}(0.20 \mathrm{~g}, 0.22 \mathrm{mmol})$ with $\mathrm{PPh}_{3}$ $(0.060 \mathrm{~g}, 0.23 \mathrm{mmol})$ in toluene $(20 \mathrm{~mL})$ afforded 12 as a yellow powder $(0.18 \mathrm{~g}, 75 \%)$. IR $\left(\mathrm{KBr}, \mathrm{cm}^{-1}\right): \tilde{v} 3361(\mathrm{~m}), 3348(\mathrm{~m}), 3054(\mathrm{w}), 2910(\mathrm{~s}), 2858(\mathrm{~m}), 1586(\mathrm{w}), 1479(\mathrm{~m}), 1436$ (s), 1378 (s), 1275 (vs), 1263 (vs), 1221 (s), 1182 (w), 1144 (vs), 1095 (m), 1030 (vs), 747 (s), 695 (s), 669 (m), 646 (vs), 637 (vs), 571 (w), 520 (s), 496 (m), 438 (m), 410 (m). ${ }^{1} \mathrm{H}$ NMR (300 MHz, $\left.\mathrm{CDCl}_{3}, 20{ }^{\circ} \mathrm{C}\right): \delta 11.67$ (s broad, $\left.3 \mathrm{H} ; \mathrm{NH}\right), 7.52-7.38(\mathrm{~m}, 9 \mathrm{H} ; o-$ and $p$ $\left.\mathrm{C}_{6} \mathrm{H}_{5}\right), 7.07-7-00\left(\mathrm{~m}, 6 \mathrm{H} ; m-\mathrm{C}_{6} \mathrm{H}_{5}\right), 1.92\left(\mathrm{~s}, 45 \mathrm{H} ; \mathrm{C}_{5} \mathrm{Me}_{5}\right) .{ }^{13} \mathrm{C}\left\{{ }^{1} \mathrm{H}\right\} \mathrm{NMR}\left(75 \mathrm{MHz}, \mathrm{CDCl}_{3}\right.$, $\left.20{ }^{\circ} \mathrm{C}\right): \delta 132.8\left(\mathrm{~d},{ }^{2} J(\mathrm{C}, \mathrm{P})=14.7 \mathrm{~Hz} ; o-\mathrm{C}_{6} \mathrm{H}_{5}\right), 131.6\left(\mathrm{~d},{ }^{1} J(\mathrm{C}, \mathrm{P})=38.8 \mathrm{~Hz} ;\right.$ ipso- $\left.\mathrm{C}_{6} \mathrm{H}_{5}\right)$, $131.0\left(\mathrm{~d},{ }^{4} J(\mathrm{C}, \mathrm{P})=1.5 \mathrm{~Hz} ; p-\mathrm{C}_{6} \mathrm{H}_{5}\right), 129.2\left(\mathrm{~d},{ }^{3} J(\mathrm{C}, \mathrm{P})=9.5 \mathrm{~Hz} ; m-\mathrm{C}_{6} \mathrm{H}_{5}\right), 121.2\left(C_{5} \mathrm{Me}_{5}\right)$, $11.7\left(\mathrm{C}_{5} M e_{5}\right)$, the $\mathrm{CF}_{3}$ carbon atom resonance was not detected. ${ }^{31} \mathrm{P}\left\{{ }^{1} \mathrm{H}\right\} \mathrm{NMR}(121 \mathrm{MHz}$, 
$\left.\mathrm{CDCl}_{3}, 20{ }^{\circ} \mathrm{C}\right): \delta 8.8\left(\mathrm{~s}, \mathrm{PPh}_{3}\right) \cdot{ }^{19} \mathrm{~F}$ NMR $\left(282 \mathrm{MHz}, \mathrm{CDCl}_{3}, 20{ }^{\circ} \mathrm{C}\right): \delta-77.9\left(\mathrm{CF}_{3}\right)$. Anal. Calcd for $\mathrm{C}_{49} \mathrm{H}_{63} \mathrm{CuF}_{3} \mathrm{~N}_{4} \mathrm{O}_{3} \mathrm{PSTi}_{3}\left(M_{w}=1083.24\right)$ : C 54.33, H 5.86, N 5.17, S 2.96. Found: C 54.17, H 6.11, N 4.92, S 3.01.

Synthesis of $\left[\left(\mathbf{P h}_{3} \mathbf{P}\right) \mathbf{A g}\left\{\left(\mu_{3}-\mathrm{NH}\right)_{3} \mathbf{T i}_{3}\left(\eta^{5}-\mathrm{C}_{5} \mathbf{M e}_{5}\right)_{3}\left(\mu_{3}-\mathrm{N}\right)\right\}\right]\left[\mathbf{O}_{3} \mathbf{S C F}_{3}\right](\mathbf{1 3})$. A $100 \mathrm{~mL}$ amber stained Schlenk flask was charged with 3 (0.15 g, $0.17 \mathrm{mmol}), \mathrm{PPh}_{3}(0.050 \mathrm{~g}, 0.19 \mathrm{mmol})$ and toluene $(20 \mathrm{~mL})$. After stirring at room temperature for $18 \mathrm{~h}$, the volatile components were removed under reduced pressure, and the resultant solid was washed with hexane (15 $\mathrm{mL})$ to afford $13 \cdot 0.5 \mathrm{C}_{7} \mathrm{H}_{8}$ as a yellow greenish solid $(0.12 \mathrm{~g}, 60 \%)$. IR $\left(\mathrm{KBr}, \mathrm{cm}^{-1}\right)$ : $\tilde{v} 3356$ (w), $3291(\mathrm{~m}), 3069$ (w), $3043(\mathrm{w}), 2908(\mathrm{~s}), 2858(\mathrm{~m}), 1603(\mathrm{w}), 1585(\mathrm{w}), 1571(\mathrm{w}), 1481$ (m), 1436 (s), 1379 (m), 1277 (vs), 1259 (vs), 1223 (s), 1147 (vs), 1097 (m), 1030 (vs), 999 (w), $761(\mathrm{~m}), 749(\mathrm{~s}), 707(\mathrm{~s}), 696(\mathrm{~s}), 654$ (vs), $636(\mathrm{vs}), 572(\mathrm{w}), 516(\mathrm{~s}), 511(\mathrm{~s}), 496(\mathrm{~m})$, 478 (w), 433 (m), 405 (m). ${ }^{1} \mathrm{H}$ NMR (300 MHz, $\mathrm{CDCl}_{3}, 20^{\circ} \mathrm{C}$ ): $\delta 12.38$ (s broad, 3H; NH), 7.53-7.40 (m, 9H; o- and $\left.p-\mathrm{C}_{6} \mathrm{H}_{5}\right), 7.16-7.08\left(\mathrm{~m}, 6 \mathrm{H} ; m-\mathrm{C}_{6} \mathrm{H}_{5}\right), 1.98\left(\mathrm{~s}, 45 \mathrm{H} ; \mathrm{C}_{5} \mathrm{Me}_{5}\right)$. ${ }^{13} \mathrm{C}\left\{{ }^{1} \mathrm{H}\right\}$ NMR $\left(75 \mathrm{MHz}, \mathrm{CDCl}_{3}, 20{ }^{\circ} \mathrm{C}\right): \delta 133.2\left(\mathrm{dd},{ }^{2} \mathrm{~J}(\mathrm{C}, \mathrm{P})=16.4 \mathrm{~Hz},{ }^{3} \mathrm{~J}(\mathrm{C}, \mathrm{Ag})=2.8\right.$ $\left.\mathrm{Hz} ; o-\mathrm{C}_{6} \mathrm{H}_{5}\right), 131.4\left(\mathrm{~d},{ }^{4} J(\mathrm{C}, \mathrm{P})=1.8 \mathrm{~Hz} ; p-\mathrm{C}_{6} \mathrm{H}_{5}\right), 131.0\left(\mathrm{dd},{ }^{1} J(\mathrm{C}, \mathrm{P})=37.0 \mathrm{~Hz},{ }^{2} J(\mathrm{C}, \mathrm{Ag})=\right.$ $3.7 \mathrm{~Hz} ;$ ipso- $\left.\mathrm{C}_{6} \mathrm{H}_{5}\right), 129.3\left(\mathrm{~d},{ }^{3} J(\mathrm{C}, \mathrm{P})=10.5 \mathrm{~Hz} ; m-\mathrm{C}_{6} \mathrm{H}_{5}\right), 121.3\left(C_{5} \mathrm{Me}_{5}\right), 11.8\left(\mathrm{C}_{5} M e_{5}\right)$, the $\mathrm{CF}_{3}$ carbon atom resonance was not detected. ${ }^{31} \mathrm{P}\left\{{ }^{1} \mathrm{H}\right\}$ NMR $\left(202 \mathrm{MHz}, \mathrm{CDCl}_{3}, 20{ }^{\circ} \mathrm{C}\right)$ : $\delta 21.0\left(\mathrm{dd},{ }^{1} J\left(\mathrm{P},{ }^{109} \mathrm{Ag}\right)=698 \mathrm{~Hz},{ }^{1} J\left(\mathrm{P},{ }^{107} \mathrm{Ag}\right)=604 \mathrm{~Hz} ; \mathrm{PPh}_{3}\right) .{ }^{19} \mathrm{~F} \mathrm{NMR}(282 \mathrm{MHz}$, $\left.\mathrm{CDCl}_{3}, 20{ }^{\circ} \mathrm{C}\right): \delta-77.9\left(\mathrm{CF}_{3}\right)$. Anal. Calcd for $\mathrm{C}_{52.5} \mathrm{H}_{67} \mathrm{AgF}_{3} \mathrm{~N}_{4} \mathrm{O}_{3} \mathrm{PSTi}_{3}\left(M_{w}=1173.64\right): \mathrm{C}$ 53.73, H 5.75, N 4.77, S 2.73. Found: C 53.61, H 5.41, N 4.47, S 2.44.

Synthesis of $\left[(\mathrm{dppm}) \mathrm{Cu}\left\{\left(\mu_{3}-\mathrm{NH}\right)_{3} \mathrm{Ti}_{3}\left(\eta^{5}-\mathrm{C}_{5} \mathrm{Me}\right)_{3}\left(\mu_{3}-\mathrm{N}\right)\right\}\right]\left[\mathrm{O}_{3} \mathrm{SCF}_{3}\right]$ (14). In a fashion similar to the preparation of $\mathbf{6}$, the treatment of $\mathbf{2} \cdot \mathrm{C}_{7} \mathrm{H}_{8}(0.30 \mathrm{~g}, 0.33 \mathrm{mmol})$ with $\mathrm{PPh}_{2} \mathrm{CH}_{2} \mathrm{PPh}_{2}(0.13 \mathrm{~g}, 0.34 \mathrm{mmol})$ in toluene $(40 \mathrm{~mL})$ for $20 \mathrm{~h}$ afforded $\mathbf{1 4} \cdot 0.5 \mathrm{C}_{7} \mathrm{H}_{8}$ as a 
yellow solid (0.33 g, 80\%). IR (KBr, cm $\left.{ }^{-1}\right): \tilde{v} 3352(\mathrm{~m}), 3289(\mathrm{~m}), 3055(\mathrm{w}), 2912(\mathrm{~s}), 2859$ (m), 1604 (w), 1586 (w), 1574 (w), 1482 (m), 1434 (s), 1379 (s), 1278 (s), 1263 (vs), 1223 (s), 1146 (vs), 1099 (m), 1031 (vs), 998 (w), 778 (m), 742 (vs), 694 (s), 647 (vs), 636 (vs), $571(\mathrm{w}), 516(\mathrm{~s}), 476(\mathrm{~m}), 434(\mathrm{~m}), 406(\mathrm{~m}) .{ }^{1} \mathrm{H}$ NMR $\left(300 \mathrm{MHz}, \mathrm{CDCl}_{3}, 20{ }^{\circ} \mathrm{C}\right): \delta 11.65$ (s broad, 3H; NH), 7.31-7.09 (m, 20H; $\left.\mathrm{C}_{6} \mathrm{H}_{5}\right), 2.81\left(\mathrm{dd},{ }^{2} J(\mathrm{H}, \mathrm{P})=6.6 \mathrm{~Hz},{ }^{2} J(\mathrm{H}, \mathrm{P})=6.6 \mathrm{~Hz}\right.$, $\left.2 \mathrm{H} ; \mathrm{CH}_{2}\right), 1.96\left(\mathrm{~s}, 45 \mathrm{H} ; \mathrm{C}_{5} \mathrm{Me}_{5}\right) .{ }^{13} \mathrm{C}\left\{{ }^{1} \mathrm{H}\right\} \mathrm{NMR}\left(75 \mathrm{MHz}, \mathrm{CDCl}_{3}, 20{ }^{\circ} \mathrm{C}\right): \delta 132.3(\mathrm{~d}$ broad, $\left.{ }^{2} J(\mathrm{C}, \mathrm{P})=17.6 \mathrm{~Hz} ; o-\mathrm{C}_{6} \mathrm{H}_{5}\right), 130.2\left(\mathrm{~s} \operatorname{broad}, p-\mathrm{C}_{6} \mathrm{H}_{5}\right), 128.8\left(\mathrm{~d} \operatorname{broad},{ }^{3} J(\mathrm{C}, \mathrm{P})=8.8 \mathrm{~Hz} ; m-\right.$ $\left.\mathrm{C}_{6} \mathrm{H}_{5}\right), 121.2\left(\mathrm{C}_{5} \mathrm{Me}_{5}\right), 29.0$ (m broad; $\left.\mathrm{CH}_{2}\right), 11.8\left(\mathrm{C}_{5} M e_{5}\right)$, the $\mathrm{CF}_{3}$ and $\mathrm{C}$-ipso of $\mathrm{C}_{6} \mathrm{H}_{5}$ carbon atom resonances were not detected. ${ }^{31} \mathrm{P}\left\{{ }^{1} \mathrm{H}\right\} \mathrm{NMR}\left(202 \mathrm{MHz}, \mathrm{CDCl}_{3}, 20{ }^{\circ} \mathrm{C}\right): \delta 2.7$ (broad; $\mathrm{Cu}-\mathrm{PPh}_{2}-\mathrm{CH}_{2}-\mathrm{PPh}_{2}$ ), -24.1 (broad; $\left.\mathrm{Cu}-\mathrm{PPh}_{2}-\mathrm{CH}_{2}-P \mathrm{Ph}_{2}\right) .{ }^{31} \mathrm{P}\left\{{ }^{1} \mathrm{H}\right\} \mathrm{NMR}(202 \mathrm{MHz}$, $\left.\mathrm{CDCl}_{3},-55^{\circ} \mathrm{C}\right): \delta 2.7\left(\mathrm{~d}\right.$ broad, $\left.{ }^{2} J(\mathrm{P}, \mathrm{P})=153.7 \mathrm{~Hz} ; \mathrm{Cu}-\mathrm{PPh}_{2}-\mathrm{CH}_{2}-\mathrm{PPh}_{2}\right),-26.1\left(\mathrm{~d},{ }^{2} J(\mathrm{P}, \mathrm{P})\right.$ $\left.=150.6 \mathrm{~Hz} ; \mathrm{Cu}-\mathrm{PPh}_{2}-\mathrm{CH}_{2}-\mathrm{PPh}_{2}\right) .{ }^{19} \mathrm{~F} \mathrm{NMR}\left(282 \mathrm{MHz}, \mathrm{CDCl}_{3}, 20{ }^{\circ} \mathrm{C}\right): \delta-77.9\left(\mathrm{CF}_{3}\right)$. Anal. Calcd for $\mathrm{C}_{59.5} \mathrm{H}_{74} \mathrm{CuF}_{3} \mathrm{~N}_{4} \mathrm{O}_{3} \mathrm{P}_{2} \mathrm{STi}_{3}\left(M_{w}=1251.42\right)$ : C 57.11, H 5.96, N 4.48, S 2.56. Found: C 57.09, H 5.82, N 4.22, S 2.44.

Synthesis of $\left[(\mathrm{dppm}) \mathrm{Ag}\left\{\left(\mu_{3}-\mathrm{NH}\right)_{3} \mathrm{Ti}_{3}\left(\eta^{5}-\mathrm{C}_{5} \mathrm{Me}_{5}\right)_{3}\left(\mu_{3}-\mathrm{N}\right)\right\}\right]\left[\mathrm{O}_{3} \mathrm{SCF}_{3}\right]$ (15). In a fashion similar to the preparation of 7, the treatment of $3(0.15 \mathrm{~g}, 0.17 \mathrm{mmol})$ with $\mathrm{PPh}_{2} \mathrm{CH}_{2} \mathrm{PPh}_{2}$ $(0.067 \mathrm{~g}, 0.17 \mathrm{mmol})$ in toluene $(15 \mathrm{~mL})$ for $18 \mathrm{~h}$ gave a yellow solid. The solid was washed with hexane $(10 \mathrm{~mL})$ to afford 15 as a yellow solid $(0.16 \mathrm{~g}, 76 \%)$. IR $\left(\mathrm{KBr}, \mathrm{cm}^{-1}\right): \tilde{v}$ 3352 (m), 3299 (m), 3054 (w), 2910 (s), 2859 (m), $1586(\mathrm{w}), 1571(\mathrm{w}), 1482(\mathrm{~m}), 1436(\mathrm{~s})$, 1380 (m), 1279 (vs), 1258 (vs), 1222 (m), 1148 (vs), 1101 (m), 1031 (vs), 999 (w), 774 (m), 742 (vs), 713 (s), 695 (s), 660 (vs), 636 (vs), $571(\mathrm{w}), 516$ (m), 478 (m), 430 (m), 404 (m). ${ }^{1} \mathrm{H}$ NMR (300 MHz, $\left.\mathrm{CDCl}_{3}, 20{ }^{\circ} \mathrm{C}\right): \delta 12.32$ (s broad, 3H; NH), 7.35-7.09 (m, 20H; $\left.\mathrm{C}_{6} \mathrm{H}_{5}\right), 2.83\left(\mathrm{dt},{ }^{3} J(\mathrm{H}, \mathrm{Ag})=6.0 \mathrm{~Hz},{ }^{2} J(\mathrm{H}, \mathrm{P})=6.0 \mathrm{~Hz}, 2 \mathrm{H} ; \mathrm{CH}_{2}\right), 1.99\left(\mathrm{~s}, 45 \mathrm{H} ; \mathrm{C}_{5} \mathrm{Me}_{5}\right)$ 
${ }^{13} \mathrm{C}\left\{{ }^{1} \mathrm{H}\right\}$ NMR $\left(75 \mathrm{MHz}, \mathrm{CDCl}_{3}, 20{ }^{\circ} \mathrm{C}\right): \delta 134.3\left(\mathrm{~m} ;\right.$ ipso- $\left.\mathrm{C}_{6} \mathrm{H}_{5}\right), 132.4\left(\mathrm{~m} ; o-\mathrm{C}_{6} \mathrm{H}_{5}\right), 130.3$ (s; $\left.p-\mathrm{C}_{6} \mathrm{H}_{5}\right), 128.9\left(\mathrm{~m} ; m-\mathrm{C}_{6} \mathrm{H}_{5}\right), 121.1\left(C_{5} \mathrm{Me}_{5}\right), 28.2$ (m broad; $\left.\mathrm{CH}_{2}\right), 11.9\left(\mathrm{C}_{5} M e_{5}\right)$, the $\mathrm{CF}_{3}$ carbon atom resonance was not detected. ${ }^{31} \mathrm{P}\left\{{ }^{1} \mathrm{H}\right\}$ NMR $\left(202 \mathrm{MHz}, \mathrm{CDCl}_{3}, 20{ }^{\circ} \mathrm{C}\right): \delta$ $2.9\left(\mathrm{dd}, J\left(\mathrm{P},{ }^{109} \mathrm{Ag}\right)=352 \mathrm{~Hz}, \mathrm{~J}\left(\mathrm{P},{ }^{107} \mathrm{Ag}\right)=305 \mathrm{~Hz} ; \mathrm{Ag}-P \mathrm{Ph}_{2}-\mathrm{CH}_{2}-P \mathrm{Ph}_{2}\right) \cdot{ }^{31} \mathrm{P}\left\{{ }^{1} \mathrm{H}\right\} \mathrm{NMR}$ $\left(202 \mathrm{MHz}, \mathrm{CD}_{2} \mathrm{Cl}_{2},-100{ }^{\circ} \mathrm{C}\right): \delta 12.2\left(\mathrm{~d}\right.$ broad, $\left.{ }^{1} J(\mathrm{P}, \mathrm{Ag})=592 \mathrm{~Hz} ; \mathrm{Ag}-\mathrm{PPh}_{2}-\mathrm{CH}_{2}-\mathrm{PPh}_{2}\right),-$ 22.1 (broad, Ag- $\left.\mathrm{PPh}_{2}-\mathrm{CH}_{2}-\mathrm{PPh}_{2}\right) .{ }^{19} \mathrm{~F}$ NMR (282 $\left.\mathrm{MHz}, \mathrm{CDCl}_{3}, 20{ }^{\circ} \mathrm{C}\right): \delta-77.9\left(\mathrm{CF}_{3}\right)$. Anal. Calcd for $\mathrm{C}_{56} \mathrm{H}_{70} \mathrm{AgF}_{3} \mathrm{~N}_{4} \mathrm{O}_{3} \mathrm{P}_{2} \mathrm{STi}_{3}\left(M_{w}=1249.67\right)$ : C 53.82, H 5.64, N 4.48, S 2.56. Found: C 53.93, H 5.56, N 4.76, S 2.42.

Reaction of 14 with 2 in a NMR Tube-Scale Experiment. A $5 \mathrm{~mm}$ valved NMR tube was charged with $14(0.008 \mathrm{~g}, 0.006 \mathrm{mmol}), 2 \cdot \mathrm{C}_{7} \mathrm{H}_{8}(0.006 \mathrm{~g}, 0.006 \mathrm{mmol})$ and chloroform- $\mathrm{d}_{1}$ $(1.00 \mathrm{~mL})$. Spectra taken after $10 \mathrm{~min}$ at room temperature showed resonance signals due to $\left[\left\{\mathrm{Cu}\left(\mu_{3}-\mathrm{NH}\right)_{3} \mathrm{Ti}_{3}\left(\eta^{5}-\mathrm{C}_{5} \mathrm{Me}\right)_{3}\left(\mu_{3}-\mathrm{N}\right)\right\}_{2}(\mu-\mathrm{dppm})\right]\left[\mathrm{O}_{3} \mathrm{SCF}_{3}\right]_{2}(\mathbf{1 6})($ ca. $60 \%)$ and those due to the starting materials. This mixture did not change upon standing at room temperature for 1 day. After addition of excess of $\mathbf{2} \cdot \mathrm{C}_{7} \mathrm{H}_{8}(0.002 \mathrm{~g}, 0.002 \mathrm{mmol})$, the spectra revealed complete consumption of $\mathbf{1 4}$ and resonances due to $\mathbf{1 6}$, toluene and the corresponding excess of 2. Complex 16 was characterized by ${ }^{1} \mathrm{H}$ and ${ }^{13} \mathrm{C}\left\{{ }^{1} \mathrm{H}\right\}$ NMR spectroscopy: ${ }^{1} \mathrm{H}$ $\operatorname{NMR}\left(300 \mathrm{MHz}, \mathrm{CDCl}_{3}, 20{ }^{\circ} \mathrm{C}\right): \delta 11.62(\mathrm{~s}$ broad, $6 \mathrm{H} ; \mathrm{NH}), 7.41\left(\mathrm{~m}, 4 \mathrm{H} ; p-\mathrm{C}_{6} \mathrm{H}_{5}\right), 7.28$ $\left(\mathrm{m}, 8 \mathrm{H} ; o-\mathrm{C}_{6} \mathrm{H}_{5}\right), 6.92\left(\mathrm{~m}, 8 \mathrm{H} ; m-\mathrm{C}_{6} \mathrm{H}_{5}\right), 3.33\left(\mathrm{t},{ }^{2} \mathrm{~J}(\mathrm{H}, \mathrm{P})=5.0 \mathrm{~Hz}, 2 \mathrm{H} ; \mathrm{CH}_{2}\right), 1.88(\mathrm{~s}, 90 \mathrm{H} ;$ $\left.\mathrm{C}_{5} \mathrm{Me}_{5}\right) \cdot{ }^{13} \mathrm{C}\left\{{ }^{1} \mathrm{H}\right\}$ NMR $\left(75 \mathrm{MHz}, \mathrm{CDCl}_{3}, 20{ }^{\circ} \mathrm{C}\right): \delta 132.6\left(\mathrm{~d},{ }^{2} J(\mathrm{C}, \mathrm{P})=14.6 \mathrm{~Hz} ; o-\mathrm{C}_{6} \mathrm{H}_{5}\right)$, $132.0\left(\mathrm{~d},{ }^{1} J(\mathrm{C}, \mathrm{P})=38.1 \mathrm{~Hz} ;\right.$ ipso- $\left.\mathrm{C}_{6} \mathrm{H}_{5}\right), 130.6\left(\mathrm{~s} ; p-\mathrm{C}_{6} \mathrm{H}_{5}\right), 129.0\left(\mathrm{~d},{ }^{3} J(\mathrm{C}, \mathrm{P})=11.0 \mathrm{~Hz} ; m-\right.$ $\left.\mathrm{C}_{6} \mathrm{H}_{5}\right), 121.3\left(\mathrm{C}_{5} \mathrm{Me}_{5}\right), 23.7$ (m broad; $\left.\mathrm{CH}_{2}\right), 11.8\left(\mathrm{C}_{5} M e_{5}\right)$, the $\mathrm{CF}_{3}$ carbon atom resonance was not detected. 
Synthesis of $\left[\left\{\mathbf{A g}\left(\mu_{3}-\mathrm{NH}\right)_{3} \mathbf{T i}_{3}\left(\eta^{5}-\mathrm{C}_{5} \mathrm{Me}_{5}\right)_{3}\left(\mu_{3}-\mathrm{N}\right)\right\}_{2}(\mu-\mathrm{dppm})\right]\left[\mathrm{O}_{3} \mathrm{SCF}_{3}\right]_{2}(17)$. A $100 \mathrm{~mL}$ amber stained Schlenk flask was charged with 3 (0.20 g, $0.23 \mathrm{mmol}), \mathrm{PPh}_{2} \mathrm{CH}_{2} \mathrm{PPh}_{2}(0.045$ $\mathrm{g}, 0.12 \mathrm{mmol})$ and toluene $(15 \mathrm{~mL})$. The reaction mixture was stirred for $15 \mathrm{~h}$ at room temperature and the volatile components were removed under reduced pressure. The resultant solid was washed with hexane $(10 \mathrm{~mL})$ and vacuum-dried to give $\mathbf{1 7} \cdot \mathrm{C}_{7} \mathrm{H}_{8}$ as a yellow solid (0.15 g, 60\%). IR (KBr, cm $\left.{ }^{-1}\right): \tilde{v} 3349$ (m), 3296 (m), 3054 (w), 2910 (s), 2859 (m), 1603 (w), $1586(\mathrm{w}), 1572$ (w), 1485 (m), 1435 (s), 1379 (s), 1276 (vs), 1259 (vs), 1222 (s), 1149 (vs), 1100 (m), 1030 (vs), 999 (w), 786 (m), 736 (vs), 694 (s), 656 (vs), 636 (vs), $571(\mathrm{w}), 516(\mathrm{~m}), 478(\mathrm{~m}), 429(\mathrm{~m}), 404(\mathrm{~m}) .{ }^{1} \mathrm{H} \mathrm{NMR}\left(300 \mathrm{MHz}, \mathrm{CDCl}_{3}, 20^{\circ} \mathrm{C}\right): \delta 12.19$ (s broad, 6H; NH), $7.45\left(\mathrm{~m}, 4 \mathrm{H} ; p-\mathrm{C}_{6} \mathrm{H}_{5}\right), 7.32\left(\mathrm{~m}, 8 \mathrm{H} ; o-\mathrm{C}_{6} \mathrm{H}_{5}\right), 7.02\left(\mathrm{~m}, 8 \mathrm{H} ; m-\mathrm{C}_{6} \mathrm{H}_{5}\right)$, 3.52 (m broad, 2H; $\left.\mathrm{CH}_{2}\right), 1.91$ (s, 90H; $\left.\mathrm{C}_{5} \mathrm{Me}_{5}\right) .{ }^{13} \mathrm{C}\left\{{ }^{1} \mathrm{H}\right\}$ NMR $\left(125 \mathrm{MHz}, \mathrm{CDCl}_{3}, 20{ }^{\circ} \mathrm{C}\right)$ : $\delta 132.7\left(\mathrm{~d},{ }^{2} J(\mathrm{C}, \mathrm{P})=15.1 \mathrm{~Hz} ; o-\mathrm{C}_{6} \mathrm{H}_{5}\right), 131.1\left(\mathrm{~s} ; p-\mathrm{C}_{6} \mathrm{H}_{5}\right), 130.8\left(\mathrm{~d},{ }^{1} J(\mathrm{C}, \mathrm{P})=34.4 \mathrm{~Hz}\right.$ ipso- $\left.\mathrm{C}_{6} \mathrm{H}_{5}\right), 129.1\left(\mathrm{~d},{ }^{3} J(\mathrm{C}, \mathrm{P})=10.4 \mathrm{~Hz} ; m-\mathrm{C}_{6} \mathrm{H}_{5}\right), 121.1\left(C_{5} \mathrm{Me}_{5}\right), 121.1\left(\mathrm{q},{ }^{1} J(\mathrm{C}, \mathrm{F})=322\right.$ $\left.\mathrm{Hz} ; \mathrm{CF}_{3}\right), 23.2$ (m broad; $\left.\mathrm{CH}_{2}\right), 11.8\left(\mathrm{C}_{5} M e_{5}\right) .{ }^{31} \mathrm{P}\left\{{ }^{1} \mathrm{H}\right\} \mathrm{NMR}\left(202 \mathrm{MHz}, \mathrm{CDCl}_{3}, 20{ }^{\circ} \mathrm{C}\right): 3.1$ $\left(\mathrm{m},{ }^{1} \mathrm{~J}(\mathrm{P}, \mathrm{Ag}) \approx 670 \mathrm{~Hz} ; \mathrm{Ag}-\mathrm{PPh}_{2}-\mathrm{CH}_{2}-\mathrm{PPh}_{2}-\mathrm{Ag}\right) .{ }^{19} \mathrm{~F} \mathrm{NMR}\left(282 \mathrm{MHz}, \mathrm{CDCl}_{3}, 20{ }^{\circ} \mathrm{C}\right): \delta-$ $77.7\left(\mathrm{CF}_{3}\right)$. Anal. Calcd for $\mathrm{C}_{94} \mathrm{H}_{126} \mathrm{Ag}_{2} \mathrm{~F}_{6} \mathrm{~N}_{8} \mathrm{O}_{6} \mathrm{P}_{2} \mathrm{~S}_{2} \mathrm{Ti}_{6}\left(M_{w}=2207.09\right): \mathrm{C}$ 51.15, H 5.75, N 5.08, S 2.90. Found: C 50.81, H 5.78, N 4.79, S 2.67.

Reaction of 2 with dppe in a NMR Tube-Scale Experiment. A $5 \mathrm{~mm}$ valved NMR tube was charged with $2 \cdot \mathrm{C}_{7} \mathrm{H}_{8}(0.009 \mathrm{~g}, 0.010 \mathrm{mmol}), \mathrm{PPh}_{2} \mathrm{CH}_{2} \mathrm{CH}_{2} \mathrm{PPh}_{2}(0.002 \mathrm{~g}, 0.005 \mathrm{mmol})$ and chloroform- $\mathrm{d}_{1}(1.00 \mathrm{~mL})$. Spectra taken after $10 \mathrm{~min}$ at room temperature showed resonance signals due to $\left[\left\{\mathrm{Cu}\left(\mu_{3}-\mathrm{NH}\right)_{3} \mathrm{Ti}_{3}\left(\eta^{5}-\mathrm{C}_{5} \mathrm{Me}_{5}\right)_{3}\left(\mu_{3}-\mathrm{N}\right)\right\}_{2}(\mu\right.$-dppe $\left.)\right]\left[\mathrm{O}_{3} \mathrm{SCF}_{3}\right]_{2}$ (18) (ca. $75 \%$ ) and those due to the starting materials. This mixture did not change upon standing at room temperature for 1 day. After addition of excess of $2 \cdot \mathrm{C}_{7} \mathrm{H}_{8}(0.003 \mathrm{~g}, 0.003$ 
mmol), the spectra revealed complete consumption of free dppe and resonances due to $\mathbf{1 8}$, toluene and the corresponding excess of 2 . Complex 18 was characterized by ${ }^{1} \mathrm{H}$ and ${ }^{13} \mathrm{C}\left\{{ }^{1} \mathrm{H}\right\}$ NMR spectroscopy: ${ }^{1} \mathrm{H}$ NMR $\left(300 \mathrm{MHz}, \mathrm{CDCl}_{3}, 20{ }^{\circ} \mathrm{C}\right.$ ): $\delta 11.56$ (s broad, $6 \mathrm{H}$; $\mathrm{NH}), 7.55\left(\mathrm{~m}\right.$ broad, $12 \mathrm{H} ; o$ - and $\left.p-\mathrm{C}_{6} \mathrm{H}_{5}\right), 7.26-7.07\left(\mathrm{~m}, 8 \mathrm{H} ; m-\mathrm{C}_{6} \mathrm{H}_{5}\right), 1.86(\mathrm{~s}, 90 \mathrm{H}$; $\mathrm{C}_{5} \mathrm{Me}_{5}$ ), 1.72 (m broad, $\left.4 \mathrm{H} ; \mathrm{CH}_{2} \mathrm{CH}_{2}\right) .{ }^{13} \mathrm{C}\left\{{ }^{1} \mathrm{H}\right\} \mathrm{NMR}\left(75 \mathrm{MHz}, \mathrm{CDCl}_{3}, 20{ }^{\circ} \mathrm{C}\right): \delta 132.2(\mathrm{~m}$; $\left.o-\mathrm{C}_{6} \mathrm{H}_{5}\right), 131.8\left(\mathrm{~d},{ }^{1} J(\mathrm{C}, \mathrm{P})=35.9 \mathrm{~Hz}\right.$; ipso- $\left.\mathrm{C}_{6} \mathrm{H}_{5}\right), 131.2\left(\mathrm{~s} ; p-\mathrm{C}_{6} \mathrm{H}_{5}\right), 129.7\left(\mathrm{~m} ; m-\mathrm{C}_{6} \mathrm{H}_{5}\right)$, $121.2\left(\mathrm{C}_{5} \mathrm{Me}_{5}\right), 23.0$ (m broad; $\left.\mathrm{CH}_{2} \mathrm{CH}_{2}\right), 11.8\left(\mathrm{C}_{5} M e_{5}\right)$, the $\mathrm{CF}_{3}$ carbon atom resonance was not detected.

Synthesis of $\left[\left\{\operatorname{Ag}(\mu 3-\mathrm{NH})_{3} \mathrm{Ti}_{3}\left(\eta^{5}-\mathrm{C}_{5} \mathrm{Me}_{5}\right)_{3}(\mu 3-\mathrm{N})\right\}_{2}(\mu-\mathrm{dppe})\right]\left[\mathrm{O}_{3} \mathrm{SCF}_{3}\right]_{2}(19)$. In a fashion similar to the preparation of 17 , treatment of $3(0.15 \mathrm{~g}, 0.17 \mathrm{mmol})$ with $\mathrm{PPh}_{2} \mathrm{CH}_{2} \mathrm{CH}_{2} \mathrm{PPh}_{2}$ $(0.035 \mathrm{~g}, 0.09 \mathrm{mmol})$ in toluene $(15 \mathrm{~mL})$ gave $19 \cdot \mathrm{C}_{7} \mathrm{H}_{8}$ as an orange solid $(0.14 \mathrm{~g}, 74 \%)$. IR $\left(\mathrm{KBr}, \mathrm{cm}^{-1}\right): \tilde{v} 3356(\mathrm{~m}), 3304(\mathrm{~m}), 3054(\mathrm{w}), 2910(\mathrm{~s}), 2859(\mathrm{~m}), 1603(\mathrm{w}), 1586(\mathrm{w}), 1571$ (w), 1485 (m), 1452 (m), 1435 (s), 1379 (m), 1263 (vs), 1222 (s), 1148 (vs), 1100 (m), $1070(\mathrm{w}), 1030$ (vs), 999 (w), 884 (w), 794 (m), 738 (vs), 696 (s), 663 (vs), 655 (vs), 636 (vs), $571(\mathrm{w}), 515$ (s), 479 (m), 464 (w), 431 (m), 405 (m). ${ }^{1} \mathrm{H}$ NMR (300 MHz, CDCl 3 , 20 $\left.{ }^{\circ} \mathrm{C}\right): \delta 12.47$ (s broad, $\left.6 \mathrm{H} ; \mathrm{NH}\right), 7.45-7.01\left(\mathrm{~m}, 20 \mathrm{H} ; \mathrm{C}_{6} \mathrm{H}_{5}\right), 2.02\left(\mathrm{~m}\right.$ broad, $\left.4 \mathrm{H} ; \mathrm{CH}_{2} \mathrm{CH}_{2}\right)$, 1.95 (s, 90H; $\left.\mathrm{C}_{5} \mathrm{Me}_{5}\right) .{ }^{13} \mathrm{C}\left\{{ }^{1} \mathrm{H}\right\} \mathrm{NMR}\left(75 \mathrm{MHz}, \mathrm{CDCl}_{3}, 20{ }^{\circ} \mathrm{C}\right): \delta 132.5\left(\mathrm{~m},{ }^{2} \mathrm{~J}(\mathrm{C}, \mathrm{P})=13.4\right.$ $\left.\mathrm{Hz} ; o-\mathrm{C}_{6} \mathrm{H}_{5}\right), 131.0\left(\mathrm{~s} ; p-\mathrm{C}_{6} \mathrm{H}_{5}\right), 129.2\left(\mathrm{~d},{ }^{3} J(\mathrm{C}, \mathrm{P})=9.8 \mathrm{~Hz} ; m-\mathrm{C}_{6} \mathrm{H}_{5}\right), 120.9\left(C_{5} \mathrm{Me}_{5}\right), 23.5$ (m broad; $\left.\mathrm{CH}_{2} \mathrm{CH}_{2}\right), 11.8\left(\mathrm{C}_{5} M e_{5}\right)$, the $\mathrm{CF}_{3}$ and $\mathrm{C}$-ipso of $\mathrm{C}_{6} \mathrm{H}_{5}$ carbon atom resonances were not detected. ${ }^{31} \mathrm{P}\left\{{ }^{1} \mathrm{H}\right\} \mathrm{NMR}\left(121 \mathrm{MHz}, \mathrm{CDCl}_{3}, 20{ }^{\circ} \mathrm{C}\right): 15.7\left(\mathrm{~m},{ }^{1} \mathrm{~J}(\mathrm{P}, \mathrm{Ag}) \approx 660 \mathrm{~Hz}\right.$; $\left.\mathrm{Ag}-\mathrm{PPh}_{2}-\mathrm{CH}_{2}-\mathrm{CH}_{2}-\mathrm{PPh}_{2}-\mathrm{Ag}\right) .{ }^{19} \mathrm{~F} \mathrm{NMR}\left(282 \mathrm{MHz}, \mathrm{CDCl}_{3}, 20{ }^{\circ} \mathrm{C}\right): \delta-77.6\left(\mathrm{CF}_{3}\right)$. Anal. Calcd for $\mathrm{C}_{95} \mathrm{H}_{128} \mathrm{Ag}_{2} \mathrm{~F}_{6} \mathrm{~N}_{8} \mathrm{O}_{6} \mathrm{P}_{2} \mathrm{~S}_{2} \mathrm{Ti}_{6}\left(M_{w}=2221.11\right)$ : C 51.37, H 5.81, N 5.04, S 2.89. Found: C 51.56, H 5.62, N 5.32, S 2.79. 
X-ray structure determination of $4,5,10,14,15$, and 18 . Crystals of complexes $4,5,10$,

14, 15, and 18 were grown as described in the Results and Discussion section, removed from the Schlenk flasks and covered with a layer of a viscous perfluoropolyether (Fomblin ${ }^{\circledR} Y$ Y). A suitable crystal was selected with the aid of a microscope, mounted on a cryoloop, and immediately placed in the low temperature nitrogen stream of the diffractometer. The intensity data sets were collected at $100 \mathrm{~K}$ for complex $\mathbf{1 0}$ or $200 \mathrm{~K}$ for the rest on a Bruker-Nonius KappaCCD diffractometer equipped with an Oxford Cryostream 700 unit. Crystallographic data for all the complexes are presented in Table 1 (see page 45).

The structures were solved, using the WINGX package, ${ }^{18}$ by direct methods (SHELXS-97) and refined by least-squares against $\mathrm{F}^{2}$ (SHELXL-97). ${ }^{19}$ Anion $\mathrm{CF}_{3} \mathrm{SO}_{3}{ }^{-}$ presented disorder in most of the structures, and this disorder was treated conventionally by using the PART tool of the SHELXL-97 program and allowing free refinement of the occupancy factors with the FVAR command. Complexes $\mathbf{4}$ and $\mathbf{5}$ crystallized with half a molecule of toluene, and both of them presented disorder for the atoms $C(1), F(1), F(2)$, $\mathrm{F}(3), \mathrm{O}(1), \mathrm{O}(2), \mathrm{O}(3)$ and $\mathrm{S}(1)$ of the triflate anions. The final values of occupancy were 51 and $49 \%$ for $\mathbf{4}$, and 52 and $48 \%$ for 5. For compound 5, DELU and SIMU restraints were also employed for the triflate anion. All non-hydrogen atoms for these compounds were anisotropically refined. The hydrogen atoms were positioned geometrically and refined using a riding model, except for those of the imido $\mathrm{NH}$ and ammonia ligands in complex 4, which were located in the difference Fourier map and refined isotropically. The atoms of the toluene molecules in both compounds were restrained with DELU instructions. 
Complex 10 crystallized with one molecule of fluorobenzene. Several attempts were made to model the solvent molecule but all were unsuccessful, therefore the Squeeze ${ }^{20}$ procedure was applied to remove its contribution to the structure factors. In addition to the disorder for the atoms $\mathrm{C}(6), \mathrm{F}(1), \mathrm{F}(2), \mathrm{F}(3), \mathrm{O}(1), \mathrm{O}(2), \mathrm{O}(3)$, and $\mathrm{S}(1)$ of the triflate anion, crystals of compound $\mathbf{1 0}$ presented disorder for the carbon atoms $\mathrm{C}(21)-\mathrm{C}(30)$ of the pentamethylcyclopentadienyl group linked to $\mathrm{Ti}(2)$, and for tert-butyl moiety of $\mathrm{CN} t \mathrm{Bu}$ $(\mathrm{C}(2), \mathrm{C}(3), \mathrm{C}(4)$ and $\mathrm{C}(5))$. These disorders were also treated by using the PART tool. The final values were 52.5 and $47.5 \%$ for the triflate anion, 55.2 and $44.8 \%$ for the pentamethylcyclopentadienyl group, and 53.1 and $46.9 \%$ for the tert-butyl group. Moreover the triflato anion was restrained with SADI and DFIX instructions. All non-hydrogen atoms for 10 were anisotropically refined, except most of the atoms for the disordered pentamethylcyclopentadienyl group $\left(\mathrm{C}(21)-\mathrm{C}(25), \mathrm{C}(28)-\mathrm{C}(30), \mathrm{C}(21)^{\prime}-\mathrm{C}(26)\right.$ ', and $\mathrm{C}(28)^{\prime}-$ $\left.\mathrm{C}(30)^{\prime}\right)$, and atoms $\mathrm{F}(2), \mathrm{O}(1), \mathrm{F}(2)^{\prime}$ ' and $\mathrm{O}(1)^{\prime}$ ' for the triflate anion, which could only be refined isotropically. The hydrogen atoms were positioned geometrically and refined using a riding model.

In the crystallographic study of compound $\mathbf{1 4}$, a molecule of hexane was found in the difference Fourier map, but it was not possible to get a chemical sensible model for it, so Squeeze ${ }^{20}$ procedure was used to remove its contribution to the structure factors. All the non-hydrogen atoms were refined anisotropically. All the hydrogen atoms were positioned geometrically and refined by using a riding model. DFIX and DELU restraints were used for the triflate anion, while the carbon atoms of the phenyl groups were also restrained with DELU instruction.

On the other hand, crystals of $\mathbf{1 5}$ presented disorder for the atoms $\mathrm{C}(2), \mathrm{F}(1), \mathrm{F}(2)$, $\mathrm{F}(3), \mathrm{O}(1), \mathrm{O}(2), \mathrm{O}(3)$ and $\mathrm{S}(1)$ of the anion triflate, and for the carbon atoms $\mathrm{C}(31)-\mathrm{C}(40)$ 
of the pentamethylcyclopentadienyl group linked to Ti(3). The PART tool was used to model both disorders. The final values were 54.4 and $45.6 \%$ for the triflate anion, and 51 and $49 \%$ for the pentamethylcyclopentadienyl group. Moreover SADI and DFIX instructions were employed to restrain the triflate anion. All non-hydrogen atoms were refined anisotropically, except $\mathrm{C}(36)$ and $\mathrm{C}(36)^{\prime}$, which were refined isotropically. All the hydrogen atoms were positioned geometrically and refined using a riding model.

Finally, compound $\mathbf{1 8}$ crystallized with two molecules of dichloromethane, and presented disorder for the atoms $\mathrm{C}(81), \mathrm{Cl}(1)$, and $\mathrm{Cl}(2)$ of the solvent molecules. The treatment of this disorder, by using the PART tool, allowed two positions for each atom to be refined with 70.1 and $29.9 \%$ of occupancy, respectively. SADI restraints were also employed for these solvent molecules. The triflate anion was restrained with DFIX and SADI instructions. All non-hydrogen atoms were refined anisotropically, except $\mathrm{C}(81)$ ', which was refined isotropically. The imido group hydrogen atoms were located in the difference Fourier map and refined isotropically, while the rest were positioned geometrically and refined using a riding model. 


\section{Results and Discussion}

The treatment of $\left[\left\{\mathrm{Ti}\left(\eta^{5}-\mathrm{C}_{5} \mathrm{Me}_{5}\right)(\mu-\mathrm{NH})\right\}_{3}\left(\mu_{3}-\mathrm{N}\right)\right]$ (1) with 0.5 equiv of $\left[\left\{\mathrm{Cu}\left(\mathrm{O}_{3} \mathrm{SCF}_{3}\right)\right\}_{2} \cdot \mathrm{C}_{7} \mathrm{H}_{8}\right]$ or 1 equiv of $\left[\mathrm{Ag}\left(\mathrm{O}_{3} \mathrm{SCF}_{3}\right)\right]$ in dichloromethane at room temperature affords the single-cube complexes $\left[\left(\mathrm{CF}_{3} \mathrm{SO}_{2} \mathrm{O}\right) \mathrm{M}\left\{\left(\mu_{3}-\mathrm{NH}\right)_{3} \mathrm{Ti}_{3}\left(\eta^{5}-\mathrm{C}_{5} \mathrm{Me}_{5}\right)_{3}\left(\mu_{3^{-}}\right.\right.\right.$ $\mathrm{N})\}]\left(\mathrm{M}=\mathrm{Cu}(\mathbf{2}),{ }^{15} \mathrm{Ag}\left(\mathbf{3}^{16}\right)\right.$ as orange or yellow solids in good yields (Scheme 1). Whereas complex $\mathbf{3}$ reacts with an additional equivalent of $\mathbf{1}$ to give the corner-shared double-cube ionic complex $\left[\mathrm{Ag}\left\{\left(\mu_{3}-\mathrm{NH}\right)_{3} \mathrm{Ti}_{3}\left(\eta^{5}-\mathrm{C}_{5} \mathrm{Me}_{5}\right)_{3}\left(\mu_{3}-\mathrm{N}\right)\right\}_{2}\right]\left[\mathrm{O}_{3} \mathrm{SCF}_{3}\right],{ }^{16}$ a product with a metalloligand to copper ratio of 2:1 could not be obtained by addition of excess 1 to complex 2. It appears that the steric bulk of the metalloligand blocks the formation of a sixcoordinate copper complex.

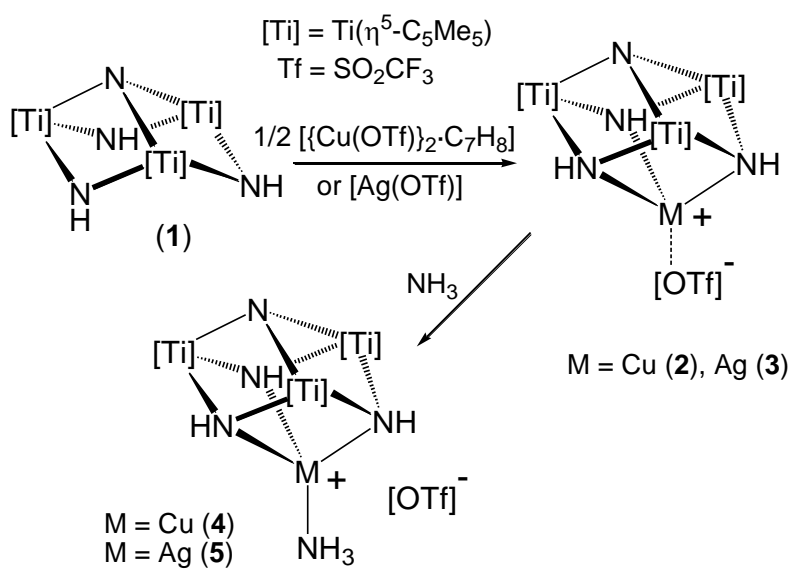

Scheme 1. Reactions of $\mathbf{2}$ and $\mathbf{3}$ with ammonia.

Despite many attempts we were not able to grow suitable single crystals of $\mathbf{2}$ and $\mathbf{3}$ for an X-ray crystal structure determination. However, toluene solutions of the complexes at -20 ${ }^{\circ} \mathrm{C}$ rendered small fractions of orange crystals of the ammonia adducts $\left[\left(\mathrm{H}_{3} \mathrm{~N}\right) \mathrm{M}\left\{\left(\mu_{3^{-}}\right.\right.\right.$ $\left.\left.\mathrm{NH})_{3} \mathrm{Ti}_{3}\left(\eta^{5}-\mathrm{C}_{5} \mathrm{Me}_{5}\right)_{3}\left(\mu_{3}-\mathrm{N}\right)\right\}\right]\left[\mathrm{O}_{3} \mathrm{SCF}_{3}\right] \cdot 0.5 \mathrm{C}_{7} \mathrm{H}_{8}\left(\mathrm{M}=\mathrm{Cu}\left(\mathbf{4} \cdot 0.5 \mathrm{C}_{7} \mathrm{H}_{8}\right), \mathrm{Ag}\left(\mathbf{5} \cdot 0.5 \mathrm{C}_{7} \mathrm{H}_{8}\right)\right) . \mathrm{We}$ suggest that the partial hydrolysis of complexes 2 and $\mathbf{3}$ could generate $\mathrm{NH}_{3}$ in the solution 
and this ammonia reacts with the remaining molecules of $\mathbf{2}$ and $\mathbf{3}$ to give the final adducts. Indeed, compounds $\mathbf{4}$ and $\mathbf{5}$ were obtained in 77 and 50\% yield respectively by treatment of 2 and 3 with excess of ammonia in toluene at room temperature. The IR (KBr) spectra of compounds 4 and 5 show several bands in the $v_{\mathrm{NH}}$ region, between 3383 and $3183 \mathrm{~cm}^{-1}$, for the imido $\mathrm{NH}$ and $\mathrm{NH}_{3}$ ligands. In addition, the IR spectra of $\mathbf{4}$ and $\mathbf{5}$ reveal one absorption at 1620 and $1617 \mathrm{~cm}^{-1}$ respectively, assignable to the $\mathrm{NH}_{3}$ bending mode. ${ }^{21,22}{ }^{1} \mathrm{H}$ and ${ }^{13} \mathrm{C}\left\{{ }^{1} \mathrm{H}\right\}$ NMR spectra in chloroform- $\mathrm{d}_{1}$ at room temperature show resonances for equivalent $\mathrm{NH}$ and $\eta^{5}-\mathrm{C}_{5} \mathrm{Me}_{5}$ groups. The $\mathrm{NH}$ resonance signals $(\delta=11.71$ and 12.33$)$ are similar to those found in the ${ }^{1} \mathrm{H}$ NMR spectra of complexes 2 and $\mathbf{3}(\delta=11.49$ and 12.25) and appear shifted toward higher field than that found for $1(\delta=13.40)$. The resonance signals for the $C_{5} \mathrm{Me}_{5}$ groups $\left(\delta=120.2\right.$ and 120.8) in the ${ }^{13} \mathrm{C}\left\{{ }^{1} \mathrm{H}\right\}$ NMR spectra of compounds $\mathbf{4}$ and $\mathbf{5}$ are also comparable to those reported for complexes $\mathbf{2}$ and $\mathbf{3}(\delta=120.2$ and 120.6) and are shifted downfield with respect to that found for $\mathbf{1}(\delta=117.1)$. These shifts are consistent with a tridentate chelate coordination of the basal $\mathrm{NH}$ groups to the copper or silver centers. ${ }^{1} \mathrm{H}$ NMR spectra of $\mathbf{4}$ and $\mathbf{5}$ reveal also one broad resonance for the ammine ligands at $\delta=2.12$ and 1.94 , respectively.

The X-ray crystal structures of $\mathbf{4}$ and $\mathbf{5}$ are presented in Figures 1 and 2, and selected distances and angles for both compounds are given in Table 2. Both compounds crystallize with a half molecule of toluene solvent per complex, and exhibit similar unit cell dimensions (see Table 1). The solid-state structures contain two $\left[\left(\mathrm{H}_{3} \mathrm{~N}\right) \mathrm{M}\left\{\left(\mu_{3}-\mathrm{NH}\right)_{3} \mathrm{Ti}_{3}\left(\eta^{5}-\mathrm{C}_{5} \mathrm{Me}_{5}\right)_{3}\left(\mu_{3}-\right.\right.\right.$ N)\}] cube-type cations associated through hydrogen bonding interactions between the ammine ligands and one oxygen atom of each trifluoromethanesulfonate anion. ${ }^{23}$ 
Figure 1. Perspective view of the dimeric disposition of $\mathbf{4}$ with thermal ellipsoids at the $50 \%$ probability level. Methyl groups of the pentamethylcyclopentadienyl ligands and the toluene molecule are omitted for clarity. Symmetry code: (a) $-\mathrm{x}$ $+1 / 2,-y+3 / 2,-z$

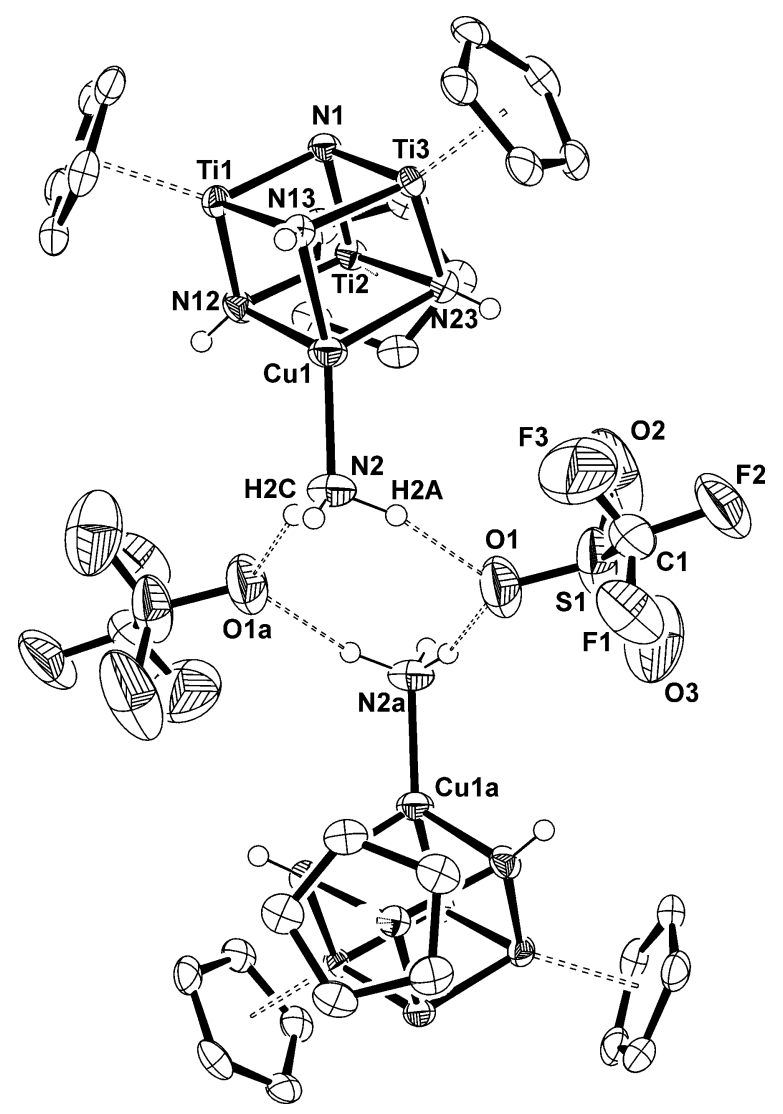


Figure 2. Perspective view of the cation in $\mathbf{5}$ with thermal ellipsoids at the $50 \%$ probability level. Hydrogen atoms of the pentamethylcyclopentadienyl ligands are omitted for clarity.

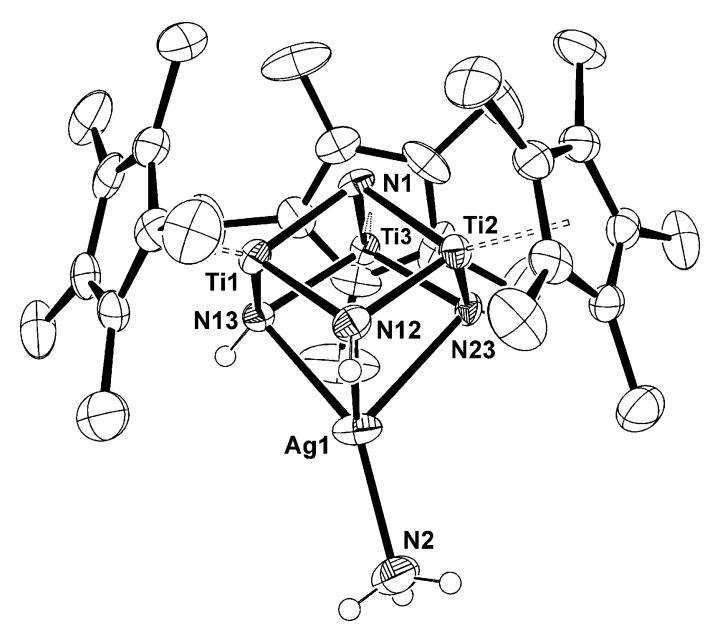


Table 2. Selected Lengths $(\AA)$ and Angles (deg) for $\mathbf{4}$ and $\mathbf{5}$.

\begin{tabular}{lcc}
\hline & $\mathrm{M}=\mathrm{Cu}(\mathbf{4})$ & $\mathrm{M}=\mathrm{Ag}(\mathbf{5})$ \\
\hline $\mathrm{M}-\mathrm{N}(2)$ & $1.989(2)$ & $2.190(4)$ \\
$\mathrm{M}-\mathrm{N}(12)$ & $2.157(2)$ & $2.503(3)$ \\
$\mathrm{M}-\mathrm{N}(13)$ & $2.089(2)$ & $2.371(3)$ \\
$\mathrm{M}-\mathrm{N}(23)$ & $2.156(2)$ & $2.497(3)$ \\
Ti(1)-N(12) & $1.951(2)$ & $1.945(3)$ \\
Ti(1)-N(13) & $1.977(2)$ & $1.976(3)$ \\
Ti(2)-N(12) & $1.963(2)$ & $1.957(4)$ \\
Ti(2)-N(23) & $1.968(2)$ & $1.973(3)$ \\
Ti(3)-N(13) & $1.974(2)$ & $1.982(3)$ \\
Ti(3)-N(23) & $1.952(2)$ & $1.950(3)$ \\
Ti-N(1) (av) & $1.939(5)$ & $1.937(1)$ \\
Ti-.Ti (av) & $2.848(6)$ & $2.853(6)$ \\
M-Ti (av) & $2.73(2)$ & $3.06(4)$ \\
& & \\
N(12)-M-N(13) & $91.8(1)$ & $79.9(1)$ \\
N(12)-M-N(23) & $90.6(1)$ & $77.9(1)$ \\
N(13)-M-N(23) & $91.8(1)$ & $80.1(1)$ \\
N(2)-M-N(12) & $114.8(1)$ & $121.1(1)$ \\
N(2)-M-N(13) & $138.0(1)$ & $150.0(1)$ \\
N(2)-M-N(23) & $118.3(1)$ & $122.8(1)$ \\
M-N-Ti (av) & $83.3(5)$ & $87(1)$ \\
N(12)-Ti(1)-N(13) & $101.8(1)$ & $106.0(1)$ \\
N(12)-Ti(2)-N(23) & $102.4(1)$ & $106.3(1)$ \\
N(13)-Ti(3)-N(23) & $101.9(1)$ & $105.8(1)$ \\
N(1)-Ti-N (av) & $86.1(2)$ & $85.7(3)$ \\
Ti-N(1)-Ti (av) & $94.5(2)$ & $94.9(2)$ \\
Ti-Nimido-Ti (av) & $92.9(3)$ & $93.2(4)$ \\
\hline
\end{tabular}

Within each cube-type cation, the metalloligand coordinates to the copper or silver centers in a tridentate chelate fashion with $\mathrm{Cu}-\mathrm{N}$ bond lengths in the range 2.089(2)-2.157(2) $\AA$ and Ag-N distances spanning 2.371(3)-2.503(3) $\AA$. These bond lengths and the N-M-N angles (average $=91.4(6)^{\circ}$ for $\mathrm{M}=\mathrm{Cu}$; average $=79(1)^{\circ}$ for $\mathrm{M}=\mathrm{Ag}$ ) are similar to those reported for four-coordinate $\mathrm{Cu}^{\mathrm{I}} / \mathrm{Ag}^{\mathrm{I}}$ ions with tris(pyrazolyl)methane ligands. ${ }^{5}$ The distorted tetrahedral geometry around copper or silver is completed by one ammine ligand showing a M-N(2) bond length $(1.989(2)$ or $2.190(4) \AA$, respectively) similar to those found in other 
copper(I)- or silver(I)-ammonia complexes..$^{22 a, 24}$ The ammine ligand is significantly tilted $\left(\mathrm{N}(2)-\mathrm{Cu}-\mathrm{N}\right.$ angles range from $114.8(1)$ to $138.0(1)^{\circ}$ and $\mathrm{N}(2)-\mathrm{Ag}-\mathrm{N}$ angles vary from 121.1(1) to $\left.150.0(1)^{\circ}\right)$ with respect to the pseudo- $C_{3}$ axis that crosses the $\mu_{3}-\mathrm{N}$ nitrido apical ligand and the group 11 metal. This distortion could be due to the hydrogen bonding interactions between the ammonia and the trifluoromethanesulfonate groups. Table 3 shows representative $\mathrm{N}(2) \cdots \mathrm{O}(1)$ distances for both complexes, and relevant $\mathrm{H} \cdots \mathrm{O}(1)$ distances found for the ammine located hydrogen atoms in $\mathbf{4 .}$

Table 3. Relevant Hydrogen Bonds ${ }^{a}$ for Compounds 4 and 5.

\begin{tabular}{llccc}
\hline & $\mathrm{D}-\mathrm{H} \cdots \mathrm{A}$ & $\mathrm{D} \cdots \mathrm{A} / \AA$ & $\mathrm{H} \cdots \mathrm{A} / \AA$ & $\mathrm{D}-\mathrm{H} \cdots \mathrm{A} /{ }^{\circ}$ \\
\hline $\mathrm{M}=\mathrm{Cu}(\mathbf{4})$ & $\mathrm{N}(2)-\mathrm{H}(2 \mathrm{~A}) \cdots \mathrm{O}(1)$ & $3.08(3)$ & $2.13(5)$ & $160(3)$ \\
& $\mathrm{N}(2)-\mathrm{H}(2 \mathrm{~A}) \cdots \mathrm{O}(1)$ & $3.16(3)$ & $2.20(5)$ & $162(3)$ \\
& $\mathrm{N}(2)-\mathrm{H}(2 \mathrm{C}) \cdots \mathrm{O}(1 \mathrm{a})$ & $3.20(3)$ & $2.42(5)$ & $152(4)$ \\
& $\mathrm{N}(2)-\mathrm{H}(2 \mathrm{C}) \cdots \mathrm{O}(1 \mathrm{a})$ & $3.23(3)$ & $2.42(5)$ & $157(4)$ \\
$\mathrm{M}=\mathrm{Ag}(\mathbf{5})$ & $\mathrm{N}(2)-\mathrm{H} \cdots \mathrm{O}(1)$ & & & \\
& $\mathrm{N}(2)-\mathrm{H} \cdots \mathrm{O}(1)$ & $3.07(4)$ & & \\
& $\mathrm{N}(2)-\mathrm{H} \cdots \mathrm{O}(1 \mathrm{a})$ & $3.09(4)$ & & \\
& $\mathrm{N}(2)-\mathrm{H} \cdots \mathrm{O}(1 \mathrm{a})$ & $3.04(4)$ & & \\
\hline
\end{tabular}

${ }^{a}$ Data for the two positions of the disordered triflate anion. Symmetry transformations: (a) $\mathrm{x}+1 / 2,-\mathrm{y}+3 / 2,-\mathrm{z} . \mathrm{A}=$ acceptor; $\mathrm{D}=$ donor. Symmetry operators apply to acceptor atoms.

Complexes $\mathbf{2}$ and $\mathbf{3}$ also react with one equivalent of pyridine (py), 2,6dimethylphenylisocyanide (CNAr), tert-butylisocyanide $(\mathrm{CN} t \mathrm{Bu})$ or triphenylphosphane in toluene at room temperature to give the analogous adducts $\left[(\mathrm{L}) \mathrm{M}\left\{\left(\mu_{3}-\mathrm{NH}\right)_{3} \mathrm{Ti}_{3}\left(\eta^{5}-\right.\right.\right.$ $\left.\left.\left.\mathrm{C}_{5} \mathrm{Me}_{5}\right)_{3}\left(\mu_{3}-\mathrm{N}\right)\right\}\right]\left[\mathrm{O}_{3} \mathrm{SCF}_{3}\right](\mathrm{L}=\mathrm{py}, \mathrm{M}=\mathrm{Cu}(\mathbf{6}), \mathrm{Ag}(\mathbf{7}) ; \mathrm{L}=\mathrm{CNAr}, \mathrm{M}=\mathrm{Cu}(\mathbf{8}), \mathrm{Ag}(\mathbf{9}) ; \mathrm{L}=$ $\left.\mathrm{CN} t \mathrm{Bu}, \mathrm{M}=\mathrm{Cu}(\mathbf{1 0}), \mathrm{Ag}(\mathbf{1 1}) ; \mathrm{L}=\mathrm{PPh}_{3}, \mathrm{M}=\mathrm{Cu}(\mathbf{1 2}), \mathrm{Ag}(\mathbf{1 3})\right)$ in good yields (60-90\%) (Scheme 2). No stable compounds were isolated on the reactions of complexes $\mathbf{2}$ and $\mathbf{3}$ with other potential ligands such as $\mathrm{COD}, \mathrm{PhCCH}, \mathrm{MeCN}$ or THF; being the starting materials 
always recovered from the reaction mixtures. Copper(I) complexes were obtained as air sensitive orange, green or yellow solids whereas silver(I) derivatives are air and light sensitive yellow or green solids and their syntheses and manipulations were carried out in the dark. Compounds 6-13 are poorly soluble in toluene or benzene but exhibit a good solubility in chloroform or dichloromethane. NMR experiments in chloroform- $\mathrm{d}_{1}$ reveal that addition of triphenylphosphane to complexes 4-11 immediately displaces $\mathrm{NH}_{3}$, py or CNR ligands from the coordination sphere of the copper and silver centers to form complexes 12 or $\mathbf{1 3 .}$

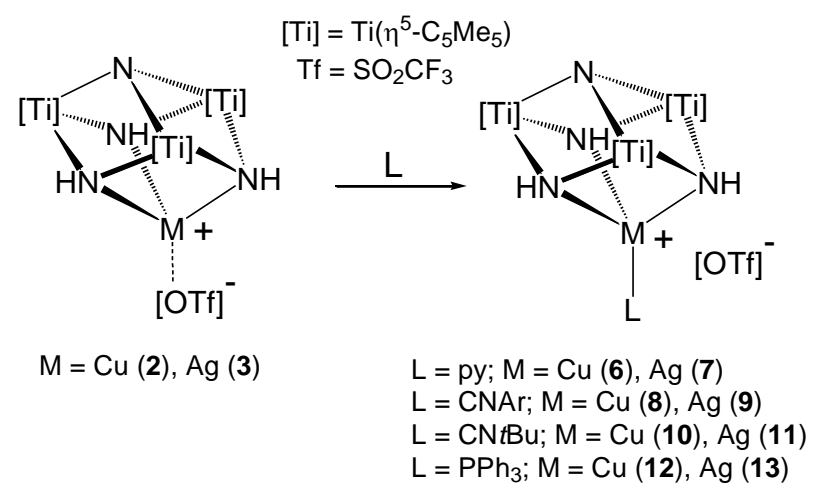

Scheme 2. Synthesis of complexes 6-13

Compounds 6-13 were characterized by spectral and analytical techniques, as well as by an X-ray crystallographic determination for $\mathbf{1 0} \cdot \mathrm{C}_{6} \mathrm{H}_{5} \mathrm{~F}$ obtained by diffusion of hexane into a saturated solution of $\mathbf{1 0}$ in fluorobenzene. IR spectra $(\mathrm{KBr})$ of complexes 6-13 show two $v_{\mathrm{NH}}$ vibrations, between 3364 and $3282 \mathrm{~cm}^{-1}$, in a similar range to the value determined for $1,{ }^{10} 3352 \mathrm{~cm}^{-1}$. Compounds 8 and 9 reveal $v(\mathrm{CN})$ stretching vibrations of the 2,6dimethylphenylisocyanide ligands at 2160 and $2177 \mathrm{~cm}^{-1}$, respectively, whereas complexes 10 and 11 reveal $v(\mathrm{CN})$ stretching vibrations of the tert-butylisocyanide ligands at 2185 and $2202 \mathrm{~cm}^{-1}$, respectively. Those values are higher than those found for the free 2,6dimethylphenylisocyanide $\left(2122 \mathrm{~cm}^{-1}\right)$ or tert-butylisocyanide $\left(2138 \mathrm{~cm}^{-1}\right)$, indicating 
predominantly $\sigma$-type M-CNR interactions. ${ }^{5 h, 25,26,27}$ The $\mathrm{CN}$ stretching vibration in complex 11 is at the same value of $2202 \mathrm{~cm}^{-1}$ for the analogue silver derivative $[(t \mathrm{BuNC}) \mathrm{Ag}\{\mathrm{HC}(3-$ $t$ Bupz $\left.\left.)_{3}\right\}\right]\left(\mathrm{O}_{3} \mathrm{SCF}_{3}\right){ }^{5 \mathrm{~b}}$ suggesting a similar donor character between the metalloligand $\mathbf{1}$ and the tris(3-tert-butylpyrazolyl)methane.

${ }^{1} \mathrm{H}$ and ${ }^{13} \mathrm{C}\left\{{ }^{1} \mathrm{H}\right\}$ NMR spectra in chloroform- $\mathrm{d}_{1}$ of complexes 6-13 at room temperature show resonances for equivalent $\mathrm{NH}$ and $\eta^{5}-\mathrm{C}_{5} \mathrm{Me}_{5}$ groups and are consistent with a $C_{3 v}$ symmetry in solution. In addition, the spectra reveal resonance signals for one pyridine, 2,6-dimethylphenylisocyanide, tert-butylisocyanide, or triphenylphosphane ligands coordinated to the copper(I) or silver(I) centers. The ${ }^{13} \mathrm{C}\left\{{ }^{1} \mathrm{H}\right\}$ NMR spectra of compounds 8 and 9 display resonances at $\delta=148.2$ and 148.9 respectively, which can be assigned to the $\mathrm{CN}$ carbon of the 2,6-dimethylphenylisocyanide ligands. ${ }^{28}$ Analogous resonance signals at $\delta=135.0$ and 139.9 in the ${ }^{13} \mathrm{C}\left\{{ }^{1} \mathrm{H}\right\}$ NMR spectra of complexes $\mathbf{1 0}$ and 11 were determined for the coordinated tert-butylisocyanide groups. ${ }^{3 \mathrm{i}, 3 \mathrm{k}}$ Those values are clearly shifted to higher field compared to those of the free 2,6-dimethylphenylisocyanide and tert-butylisocyanide ( $\delta=167.5$ and 152.3 , respectively), which has been attributed to an essentially $\sigma$-donor character of the isocyanide ligands. ${ }^{28,29}$

${ }^{31} \mathrm{P}\left\{{ }^{1} \mathrm{H}\right\}$ NMR spectra in chloroform- $\mathrm{d}_{1}$ at room temperature of compounds $\mathbf{1 2}$ and $\mathbf{1 3}$ reveal resonance signals at $\delta=8.8$ (s) and 21.0 (dd) shifted downfield with respect to that found for free $\mathrm{PPh}_{3}(\delta=-5.5 \mathrm{ppm})$. The ${ }^{31} \mathrm{P}\left\{{ }^{1} \mathrm{H}\right\} \mathrm{NMR}$ spectrum of the silver compound $\mathbf{1 3}$ at room temperature show a well-resolved pair of doublets with coupling constants ${ }^{1} J\left(\mathrm{P},{ }^{109} \mathrm{Ag}\right)=698 \mathrm{~Hz}$ and ${ }^{1} J\left(\mathrm{P},{ }^{107} \mathrm{Ag}\right)=604 \mathrm{~Hz}$, typical for complexes containing silver centers bonded to only one phosphorus atom..$^{27,30,31}$ The ratio of ${ }^{1} J\left(\mathrm{P},{ }^{109} \mathrm{Ag}\right) /{ }^{1} J\left(\mathrm{P},{ }^{107} \mathrm{Ag}\right)=$ 1.156 is consistent with the theoretical ratio of $\gamma\left({ }^{109} \mathrm{Ag}\right) / \gamma\left({ }^{107} \mathrm{Ag}\right)=1.149 .{ }^{30}$ 
The X-ray crystal structure of $\mathbf{1 0}$ is presented in Figure 3, while selected lengths and angles are given in Table 4. Compound $\mathbf{1 0}$ crystallizes with one fluorobenzene solvent molecule per ionic complex. The cation structure shows a cube-type $\left[\mathrm{CuTi}_{3} \mathrm{~N}_{4}\right]$ core with angles $\mathrm{M}-\mathrm{N}-\mathrm{M}$ and N-M-N in the range 83.2(1)-101.4(1) ${ }^{\circ}$. The coordination sphere about the copper atom is best described as distorted tetrahedral, comprising three nitrogen atoms of the tridentate organometallic ligand $\left\{\left(\mu_{3}-\mathrm{NH}\right)_{3} \mathrm{Ti}_{3}\left(\eta^{5}-\mathrm{C}_{5} \mathrm{Me}_{5}\right)_{3}\left(\mu_{3}-\mathrm{N}\right)\right\}$ and the $s p$-carbon atom of the $\mathrm{CN} t \mathrm{Bu}$ group, with $\mathrm{N}-\mathrm{Cu}-\mathrm{N}$ angles of average $91.3(3)^{\circ}$ and $\mathrm{N}-\mathrm{Cu}-\mathrm{C}(1)$ angles spanning $112.8(1)-130.1(2)^{\mathrm{o}}$. The copper-nitrogen bond lengths vary from $2.099(3)$ to $2.156(3) \AA$ (average 2.12(3) $\AA$ ) and are very similar to those found in the crystal structure of compound 4 and are slightly longer than those reported for several tris(pyrazolyl)methane complexes, where the copper atom is also coordinated to three nitrogen atoms. ${ }^{5}$ The distance from the $s p$-carbon atom $\mathrm{C}(1)$ of $\mathrm{CN} t \mathrm{Bu}$ to the copper atom of 1.861(4) $\AA$ is somewhat longer than that found for other isocyanide copper(I) complexes, such as $\left[\left(1,4-\mathrm{CNC}_{6} \mathrm{H}_{4} \mathrm{NC}\right) \mathrm{Cu}\{\mathrm{HC}(3,5-\right.$ $\left.\left.\left.\mathrm{Me}_{2} \mathrm{pz}\right)_{3}\right\}\right]\left[\mathrm{BF}_{4}\right](1.819(5) \AA)^{5 \mathrm{~h}}$ and $\left[(t \mathrm{BuNC}) \mathrm{Cu}\left\{\mathrm{HB}\left(3,5-\left(\mathrm{CF}_{3}\right)_{2} \mathrm{pz}\right)_{3}\right\}\right](1.827(6) \AA)^{3 \mathrm{i}}$. The isocyanide ligand in $\mathbf{1 0}$ is slightly bent with a $\mathrm{Cu}-\mathrm{C}(1)-\mathrm{N}(2)$ angle of $171.9(4)^{\circ}$ and possesses a normal $\mathrm{C} \equiv \mathrm{N}$ bond distance $\left(1.144(5) \AA{ }^{3}\right) .{ }^{32}$ Within the organometallic ligand, the average bond lengths and angles are similar to those determined in the free ligand $1 .{ }^{9}$ 
Figure 3. Perspective view of the cation in $\mathbf{1 0}$ with thermal ellipsoids at the $50 \%$ probability level. Hydrogen atoms are omitted for clarity.

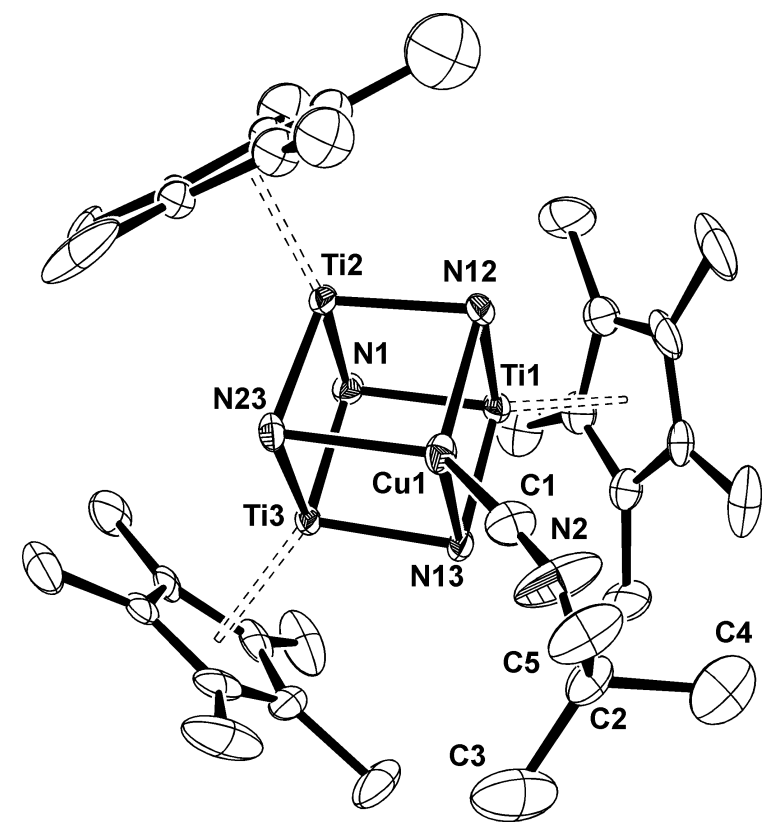

Table 4. Selected Lengths $(\AA)$ and Angles (deg) for $\mathbf{1 0 .}$

\begin{tabular}{lrlr}
\hline $\mathrm{Cu}(1)-\mathrm{C}(1)$ & $1.861(4)$ & $\mathrm{Cu}(1)-\mathrm{N}(12)$ & $2.104(3)$ \\
$\mathrm{Cu}(1)-\mathrm{N}(13)$ & $2.156(3)$ & $\mathrm{Cu}(1)-\mathrm{N}(23)$ & $2.099(3)$ \\
$\mathrm{Ti}(1)-\mathrm{N}(1)$ & $1.947(3)$ & $\mathrm{Ti}(1)-\mathrm{N}(12)$ & $1.972(3)$ \\
$\mathrm{Ti}(1)-\mathrm{N}(13)$ & $1.960(3)$ & $\mathrm{Ti}(2)-\mathrm{N}(1)$ & $1.948(3)$ \\
$\mathrm{Ti}(2)-\mathrm{N}(12)$ & $1.969(3)$ & $\mathrm{Ti}(2)-\mathrm{N}(23)$ & $1.957(3)$ \\
$\mathrm{Ti}(3)-\mathrm{N}(1)$ & $1.951(3)$ & $\mathrm{Ti}(3)-\mathrm{N}(13)$ & $1.955(3)$ \\
$\mathrm{Ti}(3)-\mathrm{N}(23)$ & $1.971(3)$ & $\mathrm{C}(1)-\mathrm{N}(2)$ & $1.144(5)$ \\
$\mathrm{Ti} \cdots \mathrm{Ti}(\mathrm{av})$ & $2.847(5)$ & $\mathrm{Cu} \cdots \mathrm{Ti}(\mathrm{av})$ & $2.731(7)$ \\
& & & \\
$\mathrm{N}(12)-\mathrm{Cu}(1)-\mathrm{N}(13)$ & $91.1(1)$ & $\mathrm{N}(13)-\mathrm{Cu}(1)-\mathrm{N}(23)$ & $91.0(1)$ \\
$\mathrm{N}(12)-\mathrm{Cu}(1)-\mathrm{N}(23)$ & $91.7(1)$ & $\mathrm{N}(12)-\mathrm{Cu}(1)-\mathrm{C}(1)$ & $130.1(2)$ \\
$\mathrm{N}(13)-\mathrm{Cu}(1)-\mathrm{C}(1)$ & $112.8(1)$ & $\mathrm{N}(23)-\mathrm{Cu}(1)-\mathrm{C}(1)$ & $128.7(2)$ \\
$\mathrm{Cu}(1)-\mathrm{C}(1)-\mathrm{N}(2)$ & $171.9(4)$ & $\mathrm{Cu}(1)-\mathrm{N}(12)-\mathrm{Ti}(1)$ & $84.3(1)$ \\
$\mathrm{Cu}(1)-\mathrm{N}(12)-\mathrm{Ti}(2)$ & $83.8(1)$ & $\mathrm{Cu}(1)-\mathrm{N}(13)-\mathrm{Ti}(1)$ & $83.2(1)$ \\
$\mathrm{Cu}(1)-\mathrm{N}(13)-\mathrm{Ti}(3)$ & $83.3(1)$ & $\mathrm{Cu}(1)-\mathrm{N}(23)-\mathrm{Ti}(2)$ & $84.2(1)$ \\
$\mathrm{Cu}(1)-\mathrm{N}(23)-\mathrm{Ti}(3)$ & $84.5(1)$ & $\mathrm{N}(12)-\mathrm{Ti}(1)-\mathrm{N}(13)$ & $101.4(1)$ \\
$\mathrm{N}(1)-\mathrm{Ti}(1)-\mathrm{N}(12)$ & $86.5(1)$ & $\mathrm{N}(1)-\mathrm{Ti}(1)-\mathrm{N}(13)$ & $86.6(1)$ \\
$\mathrm{N}(12)-\mathrm{Ti}(2)-\mathrm{N}(23)$ & $100.3(1)$ & $\mathrm{N}(1)-\mathrm{Ti}(2)-\mathrm{N}(12)$ & $86.6(1)$ \\
$\mathrm{N}(1)-\mathrm{Ti}(2)-\mathrm{N}(23)$ & $86.7(1)$ & $\mathrm{N}(13)-\mathrm{Ti}(3)-\mathrm{N}(23)$ & $101.2(1)$ \\
$\mathrm{N}(1)-\mathrm{Ti}(3)-\mathrm{N}(13)$ & $86.6(1)$ & $\mathrm{N}(1)-\mathrm{Ti}(3)-\mathrm{N}(23)$ & $86.2(1)$ \\
$\mathrm{Ti}(1)-\mathrm{N}(1)-\mathrm{Ti}(2)$ & $94.1(1)$ & $\mathrm{Ti}(1)-\mathrm{N}(1)-\mathrm{Ti}(3)$ & $93.6(1)$ \\
$\mathrm{Ti}(2)-\mathrm{N}(1)-\mathrm{Ti}(3)$ & $93.9(1)$ & $\mathrm{Ti}(1)-\mathrm{N}(12)-\mathrm{Ti}(2)$ & $92.7(1)$ \\
$\mathrm{Ti}(1)-\mathrm{N}(13)-\mathrm{Ti}(3)$ & $93.0(1)$ & $\mathrm{Ti}(2)-\mathrm{N}(23)-\mathrm{Ti}(3)$ & $93.0(1)$ \\
\hline & & &
\end{tabular}


The reactions of 2 and 3 with bisphosphanes $\mathrm{Ph}_{2} \mathrm{P}-\left(\mathrm{CH}_{2}\right)_{n}-\mathrm{PPh}_{2}(\mathrm{n}=1,2)$ in different molar ratios have been also studied (Scheme 3). Treatment of $\mathbf{2}$ and $\mathbf{3}$ with one equivalent of methylenebis(diphenylphosphane) (dppm) in toluene at room temperature led to the expected 1:1 adducts $\left[(\mathrm{dppm}) \mathrm{M}\left\{\left(\mu_{3}-\mathrm{NH}\right)_{3} \mathrm{Ti}_{3}\left(\eta^{5}-\mathrm{C}_{5} \mathrm{Me}_{5}\right)_{3}\left(\mu_{3}-\mathrm{N}\right)\right\}\right]\left[\mathrm{O}_{3} \mathrm{SCF}_{3}\right](\mathrm{M}=\mathrm{Cu}(\mathbf{1 4})$, Ag (15)) as yellow solids in good yields (80 and 76\%). The analogous reaction of 3 with a half equivalent of dppm gave the bisphosphane-bridged double-cube complex $\left[\left\{\operatorname{Ag}\left(\mu_{3}-\right.\right.\right.$ $\left.\left.\mathrm{NH})_{3} \mathrm{Ti}_{3}\left(\eta^{5}-\mathrm{C}_{5} \mathrm{Me}_{5}\right)_{3}\left(\mu_{3}-\mathrm{N}\right)\right\}_{2}(\mu-\mathrm{dppm})\right]\left[\mathrm{O}_{3} \mathrm{SCF}_{3}\right]_{2}(\mathbf{1 7})$. Compound $\mathbf{1 7} \cdot \mathrm{C}_{7} \mathrm{H}_{8}$ was isolated as a yellow solid in $60 \%$ yield in a pure form according to analytical data and NMR spectroscopy in chloroform- $\mathrm{d}_{1}$. However, the analogous treatment of the copper derivative 2 with 0.5 equiv of dppm gave a solid containing a mixture of complexes $\left[\left\{\mathrm{Cu}\left(\mu_{3^{-}}\right.\right.\right.$ $\left.\left.\mathrm{NH})_{3} \mathrm{Ti}_{3}\left(\eta^{5}-\mathrm{C}_{5} \mathrm{Me}_{5}\right)_{3}\left(\mu_{3}-\mathrm{N}\right)\right\}_{2}(\mu-\mathrm{dppm})\right]\left[\mathrm{O}_{3} \mathrm{SCF}_{3}\right]_{2}$ (16), 2 and 14, according to NMR spectroscopy in chloroform- $\mathrm{d}_{1}$. A similar mixture of compounds was obtained when in a NMR tube experiment complex 14 was treated with one equivalent of 2 . However, addition of a slight excess of $\mathbf{2}$ to this solution produced complete consumption of $\mathbf{1 4}$ allowing the characterization of $\mathbf{1 6}$ by ${ }^{1} \mathrm{H}$ and ${ }^{13} \mathrm{C}\left\{{ }^{1} \mathrm{H}\right\}$ NMR spectroscopy. 


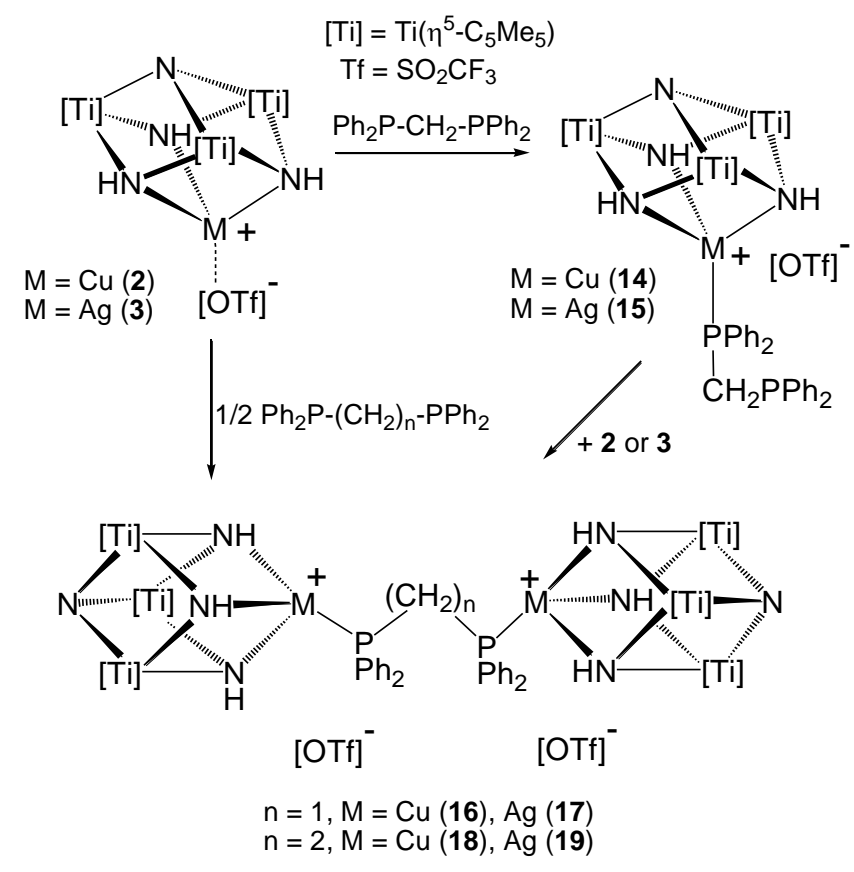

Scheme 3. Reactions of $\mathbf{2}$ and $\mathbf{3}$ with bisphosphanes.

Treatment of the silver compound $\mathbf{3}$ with a half equivalent of ethane-1,2diylbis(diphenylphosphane) (dppe) in toluene at room temperature afforded the doublecube complex $\left[\left\{\mathrm{Ag}\left(\mu_{3}-\mathrm{NH}\right)_{3} \mathrm{Ti}_{3}\left(\eta^{5}-\mathrm{C}_{5} \mathrm{Me}_{5}\right)_{3}\left(\mu_{3}-\mathrm{N}\right)\right\}_{2}(\mu-\mathrm{dppe})\right]\left[\mathrm{O}_{3} \mathrm{SCF}_{3}\right]_{2}(\mathbf{1 9})$ as an orange solid in $74 \%$ yield. However, the analogous reaction of the copper derivative 2 with dppe gave a solid containing a mixture of $\left[\left\{\mathrm{Cu}\left(\mu_{3}-\mathrm{NH}\right)_{3} \mathrm{Ti}_{3}\left(\eta^{5}-\mathrm{C}_{5} \mathrm{Me}_{5}\right)_{3}\left(\mu_{3}-\mathrm{N}\right)\right\}_{2}(\mu-\right.$ dppe) $]\left[\mathrm{O}_{3} \mathrm{SCF}_{3}\right]_{2}$ (18) (ca. 75\%) and the starting reagents, according to ${ }^{1} \mathrm{H}$ NMR spectroscopy in chloroform- $\mathrm{d}_{1}$. Fortunately, crystallization in dichloromethane/hexane at room temperature gave suitable crystals for X-ray diffraction of $18 \cdot 2 \mathrm{CH}_{2} \mathrm{Cl}_{2}$, although in low yield. NMR analysis of the crystals in chloroform- $\mathrm{d}_{1}$ revealed a mixture of $\mathbf{1 8}, \mathbf{2}$ and dppe, indicating the partial dissociation of complex 18 in solution. Upon addition of excess of 2 to this solution, the spectra revealed complete consumption of free dppe allowing the characterization of 18 by ${ }^{1} \mathrm{H}$ and ${ }^{13} \mathrm{C}\left\{{ }^{1} \mathrm{H}\right\}$ NMR spectroscopy. The decreased stability of 
double-cube complexes 16 and 18 when compared with the silver analogues 17 and 19 could be related with steric factors about the smaller copper(I) cation.

Compounds 14 and 15 were characterized by spectral and analytical methods, as well as by X-ray crystallographic determinations of crystals of $\mathbf{1 4} \cdot \mathrm{C}_{6} \mathrm{H}_{14}$ and $\mathbf{1 5}$ obtained by diffusion of hexane into solutions of $\mathbf{1 4}$ in dichloromethane or $\mathbf{1 5}$ in fluorobenzene. Spectroscopic data for $\mathbf{1 4}$ and $\mathbf{1 5}$ are similar to those of the triphenylphosphane derivatives 12 and 13. The methylene resonances of the dppm ligand in the ${ }^{1} \mathrm{H}$ NMR spectra appear as doublet of doublets at $\delta=2.81\left({ }^{2} J(\mathrm{H}, \mathrm{P})=6.6 \mathrm{~Hz},{ }^{2} J(\mathrm{H}, \mathrm{P})=6.6 \mathrm{~Hz}\right)$ for the copper complex 14 or as doublet of triplets at $\delta=2.83\left({ }^{3} J(\mathrm{H}, \mathrm{Ag})=6.0 \mathrm{~Hz},{ }^{2} J(\mathrm{H}, \mathrm{P})=6.0 \mathrm{~Hz}\right)$ for the silver compound 15. The ${ }^{13} \mathrm{C}\left\{{ }^{1} \mathrm{H}\right\}$ NMR spectra show broad signals at $\delta=29.0$ (14) or 28.2 (15) for the $\mathrm{CH}_{2}$ groups. These resonances are very close to those found in the ${ }^{1} \mathrm{H}$ and ${ }^{13} \mathrm{C}\left\{{ }^{1} \mathrm{H}\right\}$ NMR spectra of free $\operatorname{dppm}(\delta=2.78$ and 28.0, respectively).

${ }^{31} \mathrm{P}\left\{{ }^{1} \mathrm{H}\right\}$ NMR spectrum of $\mathbf{1 4}$ at room temperature in chloroform- $\mathrm{d}_{1}$ revealed broad resonance signals for coordinated $(\delta=2.7)$ and free phosphorous in the dppm ligand $(\delta=-$ 24.1). ${ }^{33}$ Upon cooling at $-55^{\circ} \mathrm{C}$ two doublets were observed with ${ }^{2} J(\mathrm{P}, \mathrm{P})$ of ca. $150 \mathrm{~Hz}$. These NMR data are consistent with a monodentate dppm ligand; in particular, the high field signal is close to that of the free dppm ligand $(\delta=-22.4)$, confirming the presence of a dangling group. However, the breadth of the resonance signals at room temperature suggests the existence of an exchange process in solution. The ${ }^{31} \mathrm{P}\left\{{ }^{1} \mathrm{H}\right\}$ NMR spectrum of the silver compound $\mathbf{1 5}$ at room temperature in chloroform- $\mathrm{d}_{1}$ shows a well-resolved pair of doublets at $\delta=-2.9$ in a fashion similar to those observed in the spectrum of the triphenylphosphane silver(I) derivative 13. However, the spectrum of $\mathbf{1 5}$ reveals silver- 
phosphorus coupling constants, $J\left(\mathrm{P},{ }^{109} \mathrm{Ag}\right)=352 \mathrm{~Hz}$ and $J\left(\mathrm{P},{ }^{107} \mathrm{Ag}\right)=305 \mathrm{~Hz}$, which are ca. the half of the values found for $13,{ }^{1} J\left(\mathrm{P},{ }^{109} \mathrm{Ag}\right)=698 \mathrm{~Hz}$ and ${ }^{1} J\left(\mathrm{P},{ }^{107} \mathrm{Ag}\right)=604 \mathrm{~Hz}$.

To investigate the coordination mode of the dppm ligand to the silver center, we studied a dichloromethane- $\mathrm{d}_{2}$ solution of $\mathbf{1 5}$ by low-temperature ${ }^{31} \mathrm{P}\left\{{ }^{1} \mathrm{H}\right\} \quad$ NMR spectroscopy in a $500 \mathrm{MHz}$ spectrometer. The room temperature ${ }^{31} \mathrm{P}\left\{{ }^{1} \mathrm{H}\right\}$ NMR spectrum of complex 15 shows one well-resolved pair of doublets by coupling with the two isotopes of silver. The simplicity of these resonances rules out the possibility of forming a doublecube structure via two $\mu$-dppm ligands since a more complicated spectra should be expected (vide infra for complexes $\mathbf{1 7}$ and 19). When cooled to $-30{ }^{\circ} \mathrm{C}$, the resonances began to broaden and, at $-100{ }^{\circ} \mathrm{C}$, gave two broad resonances at $\delta=12.2\left(\mathrm{~d},{ }^{1} J(\mathrm{P}, \mathrm{Ag}) \approx 592\right.$ $\mathrm{Hz}$ ) and $\delta=-22.1$ which coalesced at $-80{ }^{\circ} \mathrm{C}$. This nonequivalence of the phosphorus atoms in $\mathbf{1 5}$ at low temperature is similar to that observed in the NMR spectra of copper complex 14 at room temperature and is consistent with a monodentate coordination of the dppm ligand to the metal center as determined in the solid-state structures of $\mathbf{1 4}$ and $\mathbf{1 5}$ (vide infra). Fluxionality involving unidentate dppm ligands has ample precedents in the literature, ${ }^{34}$ where an intramolecular end-over-end exchange of coordinated and free phosphorous has been proposed. This also would account for the value of the coupling constants $J(\mathrm{P}, \mathrm{Ag})$ in the fast-exchange limit being the mean of ${ }^{1} J(\mathrm{P}, \mathrm{Ag})$ and ${ }^{3} J(\mathrm{P}, \mathrm{Ag}) .{ }^{33}$

The X-ray diffraction studies of $\mathbf{1 4}$ and $\mathbf{1 5}$ showed similar structures for the cationic moiety. The cation of compound $\mathbf{1 5}$ is presented in Figure 4, while selected lengths and angles for both complexes $\mathbf{1 4}$ and $\mathbf{1 5}$ are given in Table 5. While $\mathbf{1 5}$ crystallizes as a solvent-free compound, crystals of $\mathbf{1 4}$ bear one hexane solvent molecule per ionic compound. The structures confirm the azaheterometallocubane cores $\left[\mathrm{MTi}_{3} \mathrm{~N}_{4}\right]$ of the 
cationic fragments and the tridentate coordination of the metalloligand to the group 11 metals. The geometry about the copper or silver centers is best described as distorted tetrahedral, with angles of N-M-N $=90.6(4)^{\circ}$ av. $(\mathrm{Cu})$, or $79.5(3)^{\circ}$ av. $(\mathrm{Ag})$ and N-M-P(1) spanning 117.0(1)-137.5(1) ${ }^{\circ}(\mathrm{Cu})$, or 129.1(1)-134.3(1) $)^{\circ}(\mathrm{Ag})$. The copper-nitrogen bond distances in 14 range 2.105(3)-2.168(3) $\AA$ and are analogous to those determined in the structures of $\mathbf{4}$ and 10. On the other hand, the silver-nitrogen bond lengths in $\mathbf{1 5}$ range 2.422(3)-2.469(3) $\AA$ and are similar to those found for 5, and slightly longer than those reported for silver tris(pyrazolyl)methane compounds. ${ }^{5}$ The M-P(1) bond length (2.205(1) and 2.369(1) $\AA$ for copper and silver, respectively) are clearly shorter than $\mathrm{M} \cdots \mathrm{P}(2)$ distances (3.921(2) $\AA$, $\mathrm{Cu}$; and 4.054(1) $\mathrm{A}, \mathrm{Ag}$ ), accordingly to a monodentate dppm ligand coordination. The group 11 metal-phosphorous bond lengths are similar to those reported for analogous complexes with triphenylphosphane ligands, such as $\left[\left(\mathrm{Ph}_{3} \mathrm{P}\right) \mathrm{Cu}\{\mathrm{HC}(3-\right.$ $\left.\left.\mathrm{Phpz}_{3}\right\}\right]\left[\mathrm{NO}_{3}\right]^{35}$ or $\left[\left(\mathrm{Ph}_{3} \mathrm{P}\right) \mathrm{M}\left\{\mathrm{HB}\left(3,5-\mathrm{Me}_{2} \mathrm{pz}\right)_{3}\right\}\right](\mathrm{M}=\mathrm{Cu}, \mathrm{Ag}){ }^{36}$ 
Figure 4. Perspective view of the cation in complex 15 with thermal ellipsoids at the $50 \%$ probability level. Methyl groups of the pentamethylcyclopentadienyl ligands and hydrogen atoms are omitted for clarity.

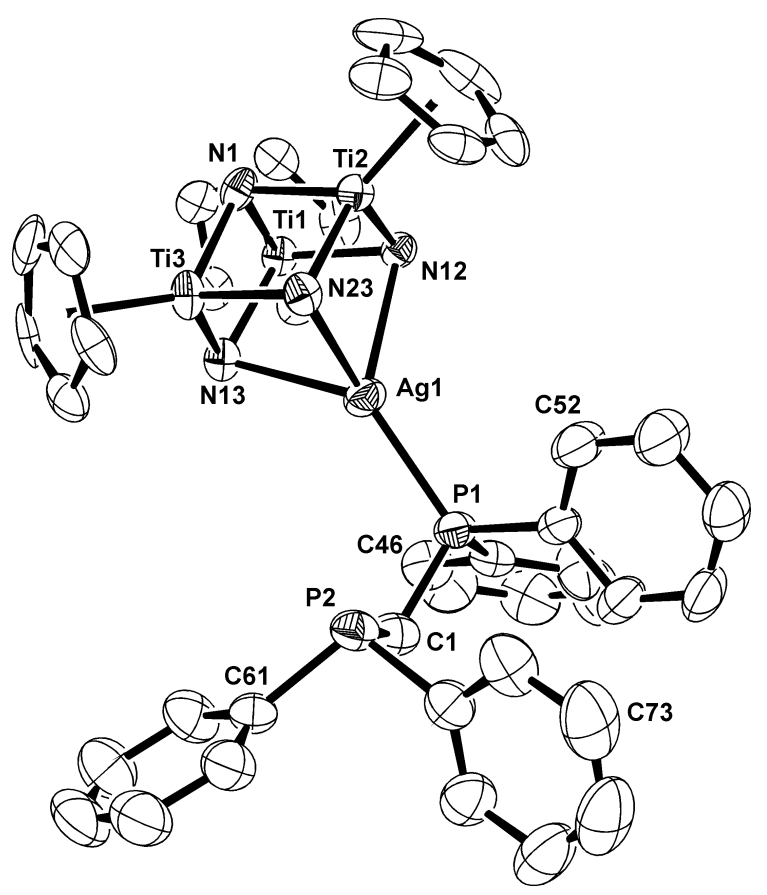


Table 5. Selected Lengths (Å) and Angles (deg) for $\mathbf{1 4}$ and $\mathbf{1 5}$.

\begin{tabular}{lcc}
\hline & $\mathrm{M}=\mathrm{Cu}(\mathbf{1 4})$ & $\mathrm{M}=\mathrm{Ag}(\mathbf{1 5})$ \\
\hline $\mathrm{M}-\mathrm{P}(1)$ & $2.205(1)$ & $2.369(1)$ \\
$\mathrm{M} \cdots \mathrm{P}(2)$ & $3.921(2)$ & $4.054(1)$ \\
$\mathrm{M}-\mathrm{N}(12)$ & $2.105(3)$ & $2.469(3)$ \\
$\mathrm{M}-\mathrm{N}(13)$ & $2.168(3)$ & $2.422(3)$ \\
$\mathrm{M}-\mathrm{N}(23)$ & $2.152(3)$ & $2.441(3)$ \\
Ti(1)-N(12) & $1.983(3)$ & $1.969(3)$ \\
Ti(1)-N(13) & $1.964(3)$ & $1.957(3)$ \\
Ti(2)-N(12) & $1.981(3)$ & $1.967(3)$ \\
Ti(2)-N(23) & $1.970(3)$ & $1.956(3)$ \\
Ti(3)-N(13) & $1.965(3)$ & $1.957(3)$ \\
Ti(3)-N(23) & $1.974(3)$ & $1.982(3)$ \\
Ti-N(1) (av) & $1.941(5)$ & $1.934(1)$ \\
Ti..Ti (av) & $2.855(2)$ & $2.850(6)$ \\
M-..Ti (av) & $2.760(5)$ & $3.060(5)$ \\
& & \\
N(12)-M-N(13) & $90.6(1)$ & $79.3(1)$ \\
N(13)-M-N(23) & $90.2(1)$ & $79.9(1)$ \\
N(12)-M-N(23) & $91.1(1)$ & $79.3(1)$ \\
P(1)-M-N(12) & $137.5(1)$ & $129.1(1)$ \\
P(1)-M-N(13) & $117.0(1)$ & $134.3(1)$ \\
P(1)-M-N(23) & $118.6(1)$ & $133.7(1)$ \\
P(1)-C(1)-P(2) & $112.0(2)$ & $111.1(2)$ \\
M-N-Ti (av) & $84.2(5)$ & $87.3(6)$ \\
N(12)-Ti(1)-N(13) & $100.7(1)$ & $105.2(1)$ \\
N(12)-Ti(2)-N(23) & $100.6(1)$ & $105.9(1)$ \\
N(13)-Ti(3)-N(23) & $102.0(1)$ & $94.8(1)$ \\
N(1)-Ti-N (av) & $86.2(2)$ & $93.0(1)$ \\
Ti-N(1)-Ti (av) & $94.7(2)$ & \\
Ti-Nimido-Ti (av) & $92.7(3)$ & \\
\hline & & \\
& & \\
\hline
\end{tabular}

Silver compounds 17 and 19 were characterized by spectral and analytical methods, while the analogous double-cube copper derivatives $\mathbf{1 6}$ and $\mathbf{1 8}$ could be only characterized by ${ }^{1} \mathrm{H}$ and ${ }^{13} \mathrm{C}\left\{{ }^{1} \mathrm{H}\right\}$ NMR spectroscopy, as well as by an X-ray crystal structure determination for $\mathbf{1 8} \cdot 2 \mathrm{CH}_{2} \mathrm{Cl}_{2}$. ${ }^{1} \mathrm{H}$ and ${ }^{13} \mathrm{C}\left\{{ }^{1} \mathrm{H}\right\}$ NMR spectra of complexes 16-19 in chloroform- $\mathrm{d}_{1}$ reveal resonances for equivalent $\mathrm{NH}$ and $\eta^{5}-\mathrm{C}_{5} \mathrm{Me}_{5}$ groups. In addition, the spectra show resonance signals for one dppm or dppe ligand per two $\left\{\mathrm{M}\left(\mu_{3}-\mathrm{NH}\right)_{3} \mathrm{Ti}_{3}\left(\eta^{5}-\right.\right.$ 
$\left.\left.\mathrm{C}_{5} \mathrm{Me}_{5}\right)_{3}\left(\mu_{3}-\mathrm{N}\right)\right\}$ moieties. The methylene resonances of the dppm ligand in the ${ }^{1} \mathrm{H}$ NMR spectra appear as a triplet at $\delta=3.33\left({ }^{2} J(\mathrm{H}, \mathrm{P})=5.0 \mathrm{~Hz}\right)$ for $\mathbf{1 6}$ or a multiplet centered at $\delta=$ 3.52 for the silver compound $\mathbf{1 7}$, clearly shifted downfield with respect to those found in complexes 14 and 15 ( $\delta=2.81$ and 2.83, respectively) and free dppm $(\delta=2.78)$. However, the resonances for the $\mathrm{CH}_{2}$ groups, $\delta=23.7$ (16) and $23.2(\mathbf{1 7})$, in the ${ }^{13} \mathrm{C}\left\{{ }^{1} \mathrm{H}\right\}$ NMR spectra are shifted upfield when compared with those observed in the spectra of $\mathbf{1 4}(\delta=$ 29.0), $15(\delta=28.2)$, and free dppm $(\delta=28.0)$. On the other hand, the ethylene resonances of the dppe ligand in the ${ }^{1} \mathrm{H}$ and ${ }^{13} \mathrm{C}\left\{{ }^{1} \mathrm{H}\right\}$ NMR spectra of complexes $\mathbf{1 8}$ and $\mathbf{1 9}$ appear as broad signals at chemical shifts $\left(\delta_{\mathrm{H}}=1.72\right.$ and $2.02, \delta_{\mathrm{C}}=23.0$ and 23.5) close to those of free dppe $\left(\delta_{\mathrm{H}}=2.07, \delta_{\mathrm{C}}=23.8\right)$.

The ${ }^{31} \mathrm{P}\left\{{ }^{1} \mathrm{H}\right\}$ NMR spectra in chloroform- $\mathrm{d}_{1}$ at room temperature of the silver compounds 17 and 19 consist of a multiplet resonances centered at $\delta=3.1$ and 15.7, respectively, which are shifted downfield with respect to that found for free dppm ( $\delta=$ -22.4) and dppe ( $\delta=-12.6)$. The complex spectral pattern for these compounds is due to overlapping spectra of isotopomers containing ${ }^{109} \mathrm{Ag}^{109} \mathrm{Ag},{ }^{109} \mathrm{Ag}{ }^{107} \mathrm{Ag}$, and ${ }^{107} \mathrm{Ag}^{107} \mathrm{Ag}$ pairs in the dinuclear $\mathrm{Ag}_{2}(\mu-\mathrm{dppm})$ or $\mathrm{Ag}_{2}(\mu$-dppe) structures. Very similar spectra have been reported and analyzed in detail for several dinuclear silver compounds with bridging dppm ligands. ${ }^{37}$ The separation of the major lines of the spectra allows to estimate coupling constants ${ }^{1} J(\mathrm{P}, \mathrm{Ag}) \approx 670 \mathrm{~Hz}$ for 17 and ${ }^{1} J(\mathrm{P}, \mathrm{Ag}) \approx 660 \mathrm{~Hz}$ for 19, which are comparable to those found in the spectra of $\mathbf{1 3}$ and other complexes containing silver centers bonded to only one phosphorus atom. ${ }^{30}$

Compound 18 crystallizes with two dichloromethane solvent molecules per ionic compound. The structure of the cationic fragment shows two cube-type cores $\left[\mathrm{CuTi}_{3} \mathrm{~N}_{4}\right]$ 
with a dppe ligand linking the two copper atoms (Figure 5). Selected distances and angles of the cation are given in Table 6. The complex has an inversion center located between the two methylene carbon atoms of the dppe ligand. The coordination geometry about each of the two copper atoms could be described as distorted tetrahedral, comprising three nitrogen atoms of the metalloligand and one phosphorous atom of the dppe, with angles $\mathrm{N}-\mathrm{Cu}(1)-\mathrm{N}$ of average $89.8(3)^{\circ}$ and angles $\mathrm{P}(1)-\mathrm{Cu}(1)-\mathrm{N}$ in the range of $114.1(1)-134.2(1)^{\circ}$. The copper-nitrogen bond lengths $(2.132(3)-2.166(3) \AA)$ are similar to those found in complexes 4, 10, and 14, and the $\mathrm{Cu}(1)-\mathrm{P}(1)$ distance $(2.219(1) \AA)$ is slightly longer than that observed in 14. The bond distances and angles around the copper center in $\mathbf{1 8}$ compare well with those found in the crystal structures of dinuclear copper(I) complexes containing one bridging dppe ligand. ${ }^{38}$

Figure 5. Perspective view of the cation in $\mathbf{1 8}$ with thermal ellipsoids at the $50 \%$ probability level. Methyl groups of the pentamethylcyclopentadienyl ligands and hydrogen atoms are omitted for clarity. Symmetry code: (a) $-\mathrm{x}, 2-\mathrm{y}, 1-\mathrm{z}$.

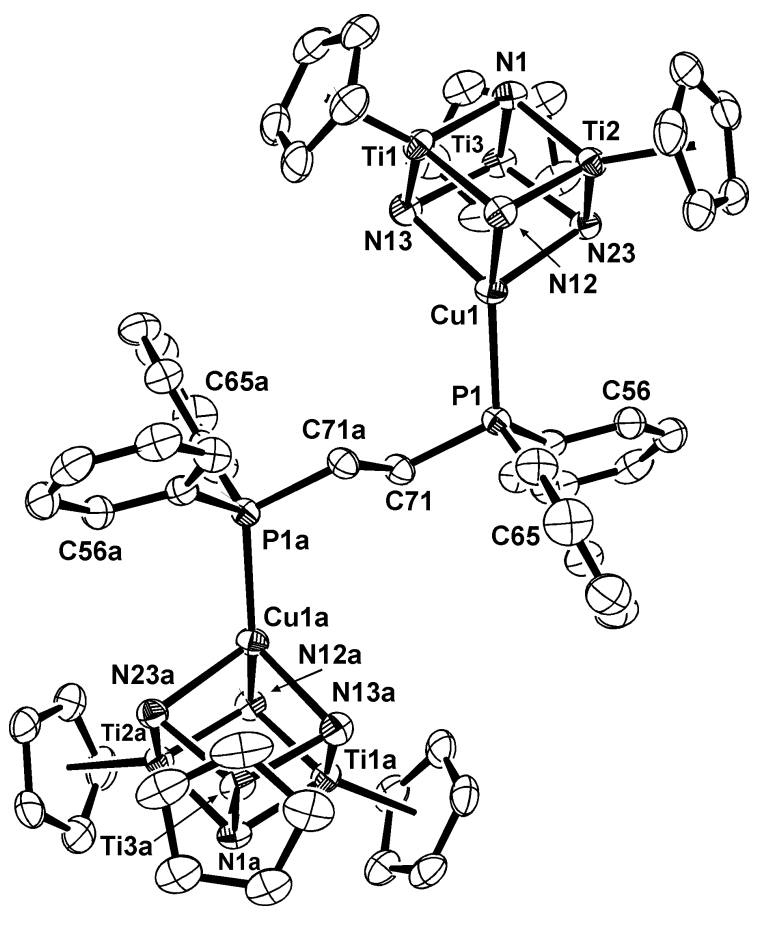


Table 6. Selected Lengths $(\AA)$ and Angles (deg) for $\mathbf{1 8 .}$

\begin{tabular}{lclc}
\hline $\mathrm{Cu}(1)-\mathrm{P}(1)$ & $2.219(1)$ & $\mathrm{Cu}(1)-\mathrm{N}(12)$ & $2.132(3)$ \\
$\mathrm{Cu}(1)-\mathrm{N}(13)$ & $2.159(3)$ & $\mathrm{Cu}(1)-\mathrm{N}(23)$ & $2.166(3)$ \\
$\mathrm{Ti}(1)-\mathrm{N}(12)$ & $1.965(3)$ & $\mathrm{Ti}(1)-\mathrm{N}(13)$ & $1.973(3)$ \\
$\mathrm{Ti}(2)-\mathrm{N}(12)$ & $1.971(3)$ & $\mathrm{Ti}(2)-\mathrm{N}(23)$ & $1.964(3)$ \\
$\mathrm{Ti}(3)-\mathrm{N}(13)$ & $1.977(3)$ & $\mathrm{Ti}(3)-\mathrm{N}(23)$ & $1.966(3)$ \\
$\mathrm{Ti}-\mathrm{N}(1)(\mathrm{av})$ & $1.943(6)$ & $\mathrm{Ti} \cdots \mathrm{Ti}(\mathrm{av})$ & $2.860(9)$ \\
$\mathrm{Cu}(1) \cdots \mathrm{Ti}$ (av) & $2.78(1)$ & & \\
& & & \\
$\mathrm{N}(12)-\mathrm{Cu}(1)-\mathrm{N}(13)$ & $89.4(1)$ & $\mathrm{N}(13)-\mathrm{Cu}(1)-\mathrm{N}(23)$ & $89.8(1)$ \\
$\mathrm{N}(12)-\mathrm{Cu}(1)-\mathrm{N}(23)$ & $90.2(1)$ & $\mathrm{P}(1)-\mathrm{Cu}(1)-\mathrm{N}(12)$ & $134.2(1)$ \\
$\mathrm{P}(1)-\mathrm{Cu}(1)-\mathrm{N}(13)$ & $126.5(1)$ & $\mathrm{P}(1)-\mathrm{Cu}(1)-\mathrm{N}(23)$ & $114.1(1)$ \\
$\mathrm{Cu}(1)-\mathrm{N}-\mathrm{Ti}(\mathrm{av})$ & $84.6(6)$ & $\mathrm{N}(12)-\mathrm{Ti}(1)-\mathrm{N}(13)$ & $100.1(1)$ \\
$\mathrm{N}(12)-\mathrm{Ti}(2)-\mathrm{N}(23)$ & $101.4(1)$ & $\mathrm{N}(13)-\mathrm{Ti}(3)-\mathrm{N}(23)$ & $101.5(1)$ \\
$\mathrm{N}(1)-\mathrm{Ti}-\mathrm{N}$ (av) & $86.0(5)$ & $\mathrm{Ti}-\mathrm{N}(1)-\mathrm{Ti}(\mathrm{av})$ & $94.8(3)$ \\
$\mathrm{Ti}-\mathrm{N}_{\text {imido-Ti (av) }}$ & $93.1(5)$ & & \\
\hline
\end{tabular}

\section{Conclusion}

We have presented the systematic syntheses of a series of cationic copper(I) and silver(I) complexes incorporating the neutral tridentate imido-nitrido titanium metalloligand 1. These derivatives $\left[(\mathrm{L}) \mathrm{M}\left\{\left(\mu_{3}-\mathrm{NH}\right)_{3} \mathrm{Ti}_{3}\left(\eta^{5}-\mathrm{C}_{5} \mathrm{Me}_{5}\right)_{3}\left(\mu_{3}-\mathrm{N}\right)\right\}\right]\left[\mathrm{O}_{3} \mathrm{SCF}_{3}\right]$ were prepared by displacement of the labile trifluoromethanesulfonate ligand in complexes $\left[\left(\mathrm{CF}_{3} \mathrm{SO}_{2} \mathrm{O}\right) \mathrm{M}\left\{\left(\mu_{3}-\mathrm{NH}\right)_{3} \mathrm{Ti}_{3}\left(\eta^{5}-\mathrm{C}_{5} \mathrm{Me}_{5}\right)_{3}\left(\mu_{3}-\mathrm{N}\right)\right\}\right] \quad(\mathrm{M}=\mathrm{Cu} \quad(2), \quad \mathrm{Ag}(3))$ with more nucleophilic ammine, pyridine, isocyanide or phosphane ligands. Whereas the reaction of 2 or 3 with one equivalent of dppm led to single-cube complexes $\left[(\mathrm{dppm}) \mathrm{M}\left\{\left(\mu_{3}-\mathrm{NH}\right)_{3} \mathrm{Ti}_{3}\left(\eta^{5}-\right.\right.\right.$ $\left.\left.\left.\mathrm{C}_{5} \mathrm{Me}_{5}\right)_{3}\left(\mu_{3}-\mathrm{N}\right)\right\}\right]\left[\mathrm{O}_{3} \mathrm{SCF}_{3}\right]$ containing monodentate bisphosphane ligands, the analogous treatment with a half equivalent of dppm or dppe afforded double-cube systems where a bidentate bisphosphane ligand bridge two $\left[\left\{\mathrm{M}\left(\mu_{3}-\mathrm{NH}\right)_{3} \mathrm{Ti}_{3}\left(\eta^{5}-\mathrm{C}_{5} \mathrm{Me}_{5}\right)_{3}\left(\mu_{3}-\mathrm{N}\right)\right]\right.$ cations. 
Acknowledgments. We thank the Spanish MEC (CTQ2008-00061/BQU) and Factoría de Cristalización (CONSOLIDER-INGENIO 2010) for financial support of this research. N.M.-E. thanks the MEC for a doctoral fellowship.

Supporting Information Available: X-ray crystallographic files in CIF format for complexes $4, \mathbf{5}, \mathbf{1 0}, \mathbf{1 4}, \mathbf{1 5}$, and $\mathbf{1 8}$. This material is available free of charge via the Internet at http://pubs.acs.org.

\section{Notes}

The authors declare no competing financial interest.

\section{References}

(1) For general references, see: (a) Trofimenko, S. Scorpionates: The Coordination Chemistry of Polypyrazolylborate Ligands; Imperial College: London, UK, 1999. (b) Pettinari, C.; Santini, C. in Comprehensive Coordination Chemistry II; McCleverty, J. A., Meyer, T. J., Ed.; Elsevier: Oxford, UK, 2004; Vol. 1, pp. 159-210. (c) Pettinari, C. Scorpionates II: Chelating Borate Ligands; Imperial College: London, UK, 2008. (d) Trofimenko, S. Chem Rev. 1993, 93, 943-980. (e) Kitajima, N.; Tolman, B. W. Prog. Inorg. Chem. 1995, 43, 419531.

(2) Pettinari, C.; Cingolani, A.; Gioia Lobbia, G.; Marchetti, F.; Martini, D.; Pellei, M.; Pettinari, R.; Santini, C. Polyhedron, 2004, 23, 451-469.

(3) For a selection of copper(I) and silver(I) complexes with tris(pyrazolyl)borate ligands, see: (a) Churchill, M. R.; DeBoer, B. G.; Rotella, F. J.; Abu Salah, O. M.; Bruce, M. I. Inorg. Chem. 1975, 14, 2051-2056. (b) Thompson, J. S.; Harlow, R. L.; Whitney, J. F. J. Am. Chem. Soc. 1983, 105, 3522-3527. (c) Kitajima, N.; Koda, T.; Hashimoto, S.; Kitagawa, T.; Moro-oka, Y. J. Am. Chem. Soc. 1991, 113, 5664-5671. (d) Kitajima, N.; Fujisawa, K.; 
Fujimoto, C.; Moro-oka, Y.; Hashimoto, S.; Kitagawa, T.; Toriumi, K.; Tatsumi, K.; Nakamura, A. J. Am. Chem. Soc. 1992, 114, 1277-1291. (e) Carrier, S. M.; Ruggiero, C. E.; Tolman, W. B. J. Am. Chem. Soc. 1992, 114, 4407-4408. (f) Carrier, S. M.; Ruggiero, C. E.; Houser, R. P.; Tolman, W. B. Inorg. Chem. 1993, 32, 4889-4899. (g) Dias, H. V. R.; Jin, W. J. Am. Chem. Soc. 1995, 117, 11381-11382. (h) Dias, H. V. R.; Jin, W. Inorg. Chem. 1996, 35, 267-268. (i) Dias, H. V. R.; Lu, H.-L.; Gorden, J. D.; Jin, W. Inorg. Chem. 1996, 35, 2149-2151. (j) Dias, H. V. R.; Jin, W.; Kim, H.-J.; Lu, H.-L. Inorg. Chem. 1996, 35, 2317-2328. (k) Dias, H. V. R.; Wang, Z.; Jin, W. Inorg. Chem. 1997, 36, 6205-6215. (1) Imai, S.; Fujisawa, K.; Kobayashi, T.; Shirasawa, N.; Fujii, H.; Yoshimura, T.; Kitajima, N.; Moro-oka, Y. Inorg. Chem. 1998, 37, 3066-3070. (m) Santini, C.; Gioia Lobbia, G.; Pellei, M.; Pettinari, C.; Valle, G.; Calogero, S. Inorg. Chim. Acta 1998, 282, 1-9.

(4) For general references, see: (a) Reger, D. L. Comments Inorg. Chem. 1999, 21, 1-28. (b) Bigmore, H. R.; Lawrence, S. C.; Mountford, P.; Tredget, C. S. Dalton Trans. 2005, 635651. (c) Pettinari, C.; Pettinari, R. Coord. Chem. Rev. 2005, 249, 525-543.

(5) For a selection of copper(I) and silver(I) complexes with tris(pyrazolyl)methane ligands, see: (a) Reger, D. L.; Collins, J. E.; Rheingold, A. L.; Liable-Sands, L. M. Organometallics 1996, 15, 2029-2032. (b) Reger, D. L.; Collins, J. E.; Rheingold, A. L.; Liable-Sands, L. M.; Yap, G. P. A. Organometallics 1997, 16, 349-353. (c) Cvetkovic, M.; Batten, S. R.; Moubaraki, B.; Murray, K. S.; Spiccia, L. Inorg. Chim. Acta 2001, 324, 131-140. (d) Cingolani, A.; Effendy; Martini, D.; Pellei, M.; Pettinari, C.; Skelton, B. W.; White, A. H. Inorg. Chim. Acta 2002, 328, 87-95. (e) Reger, D. L.; Semeniuc, R. F.; Smith, M. D. Eur. J. Inorg. Chem. 2003, 3480-3494. (f) Fujisawa, K.; Ono, T.; Ishikawa, Y.; Amir, N.; Miyashita, Y.; Okamoto, K.; Lehnert, N. Inorg. Chem. 2006, 45, 1698-1713. (g) Reger, D. 
L.; Semeniuc, R. F.; Little, C. A.; Smith, M. D. Inorg. Chem. 2006, 45, 7758-7769. (h)

Hsu, S. C. N.; Chen, H. H. Z.; Lin, I.-J.; Liu, J.-J.; Chen, P.-Y. J. Organomet. Chem. 2007, 692, 3676-3684.

(6) For a general reference and selected examples of copper(I) and silver(I) complexes with 1,4,7-triazacyclononane ligands, see: (a) Chaudhuri, P.; Wieghardt, K. Prog. Inorg. Chem. 1987, 35, 329-436. (b) Stockheim, C.; Wieghardt, K.; Nuber, B.; Weiss, J.; Flörke, U.; Haupt, H.-J. J. Chem. Soc., Dalton Trans. 1991, 1487-1490. (c) Mahapatra, S.; Halfen, J. A.; Wilkinson, E. C.; Que, L., Jr.; Tolman, W. B. J. Am. Chem. Soc. 1994, 116, 9785-9786. (d) Kujime, M.; Kurahashi, T.; Tomura, M.; Fujii, H. Inorg. Chem. 2007, 46, 541-551.

(7) For a selection of copper(I) complexes with 1,3,5-triazacyclohexane ligands, see: (a) Köhn, R. D.; Seifert, G.; Kociok-Köhn, G. Chem. Ber. 1996, 129, 1327-1333. (b) Köhn, R. D.; Pan, Z.; Mahon, M. F.; Kociok-Köhn, G. Chem Commun. 2003, 1272-1273. (c) Köhn, R. D.; Pan, Z.; Mahon, M. F.; Kociok-Köhn, G. Dalton Trans. 2003, 2269-2275. (d) Köhn, R. D.; Pan, Z.; Haufe, M.; Kociok-Köhn, G. Dalton Trans. 2005, 2793-2797. (e) Köhn, R. D.; Tomás Laudo, L.; Pan, Z.; Speiser, F.; Kociok-Köhn, G. Dalton Trans. 2009, 4556-4568.

(8) For general references of metal nitrido complexes, see: (a) Nugent, W. A.; Mayer, J. M. Metal-Ligand Multiple Bonds; Wiley: New York, 1988. (b) Dehnicke, K.; Strähle, J. Angew. Chem. Int. Ed. Engl. 1981, 20, 413-426. (c) Dehnicke, K.; Strähle, J. Angew. Chem. Int. Ed. Engl. 1992, 31, 955-978. (d) Dehnicke, K.; Weller, F.; Strähle J. Chem. Soc. Rev. 2001, 30, 125-135.

(9) Roesky, H. W.; Bai, Y.; Noltemeyer, M. Angew. Chem. Int. Ed. Engl. 1989, 28, 754-755.

(10) Abarca, A.; Gómez-Sal, P.; Martín, A.; Mena, M.; Poblet, J.-M.; Yélamos, C. Inorg. Chem. 2000, 39, 642-651. 
(11) Haack, K. J.; Goddard, R.; Pörschke, K. R. J. Am. Chem. Soc. 1997, 119, 7992-7999.

(12) (a) Abarca, A.; Galakhov, M.; Gómez-Sal, P.; Martín, A.; Mena, M.; Poblet, J.-M.; Santamaría, C.; Sarasa, J. P. Angew. Chem. Int. Ed. 2000, 39, 534-537. (b) Abarca, A.; Martín, A.; Mena, M.; Yélamos, C. Angew. Chem. Int. Ed. 2000, 39, 3460-3463. (c) Freitag, K.; Gracia, J.; Martín, A.; Mena, M.; Poblet, J.-M.; Sarasa, J. P.; Yélamos, C. Chem. Eur. J. 2001, 7, 3644-3651. (d) Abarca, A.; Galakhov, M. V.; Gracia, J.; Martín, A.; Mena, M.; Poblet, J.-M.; Sarasa, J. P.; Yélamos, C. Chem. Eur. J. 2003, 9, 2337-2346. (e) Martín, A.; Martínez-Espada, N.; Mena, M.; Pérez-Redondo, A.; Yélamos, C. Inorg. Chem. 2006, 45, 6901-6911. (f) Martínez-Espada, N.; Mena, M.; Mosquera, M. E. G.; PérezRedondo, A.; Yélamos, C. Organometallics 2010, 29, 6732-6738.

(13) (a) Caballo, J.; García-Castro, M.; Martín, A.; Mena, M.; Pérez-Redondo, A.; Yélamos, C. Inorg. Chem. 2008, 47, 7077-7079. (b) Caballo, J.; García-Castro, M.; Martín, A.; Mena, M.; Pérez-Redondo, A.; Yélamos, C. Inorg. Chem. 2011, 50, 6798-6808.

(14) (a) García-Castro, M.; Martín, A.; Mena, M.; Yélamos, C. Organometallics 2004, 23, 1496-1500. (b) García-Castro, M.; Gracia, J.; Martín, A.; Mena, M.; Poblet. J.-M.; Sarasa, J. P.; Yélamos, C. Chem. Eur. J. 2005, 11, 1030-1041.

(15) Carbó, J. J.; Martínez-Espada, N.; Mena, M.; Mosquera, M. E. G.; Poblet, J.-M.; Yélamos, C. Chem. Eur. J. 2009, 15, 11619-11631.

(16) Martín, A.; Martínez-Espada, N.; Mena, M.; Yélamos, C. Chem. Commun. 2007, 29832985.

(17) Lawrance, G. A. Chem. Rev. 1986, 86, 17-33.

(18) Farrugia, L. J. J. Appl. Crystallogr. 1999, 32, 837-838.

(19) Sheldrick, G. M. Acta Crystallogr. 2008, A64, 112-122. 
(20) SQUEEZE; Sluis, P. van der; Spek, A. L. Acta Crystallogr. 1990, A46, 194-201.

(21) Nakamoto, K. Infrared and Raman Spectra of Inorganic and Coordination Compounds, 5th ed.; Wiley: New York, 1997.

(22) (a) Smith, G.; Reddy, A. N.; Byriel, K. A.; Kennard, C. H. L. J. Chem. Soc., Dalton Trans. 1995, 3565-3570. (b) Whitcomb, D. R.; Rogers, R. D. Polyhedron 1997, 16, 863-868.

(23) Steiner, T. Angew. Chem. Int. Ed. 2002, 41, 48-76.

(24) (a) Falvello, L. R.; Pascual, I.; Tomás, M.; Urriolabeitia, E. P. J. Am. Chem. Soc. 1997, 119, 11894-11902. (b) Mironov, Y. V.; Efremova, O. A.; Naumov, D. Y.; Sheldrick, W. S.; Fedorov, V. E. Eur. J. Inorg. Chem. 2003, 2591-2595. (c) Whitcomb, D. R.; Rajeswaran, M. Inorg. Chim. Acta 2008, 361, 1357-1362, and references therein.

(25) Cotton, F. A.; Zingales, F. J. Am. Chem. Soc. 1961, 83, 351-355.

(26) Effendy; Gioia Lobbia, G.; Pettinari, C.; Santini, C.; Skelton, B. W.; White, A. H. Inorg. Chim. Acta 2000, 298, 146-153.

(27) Dias, H. V. R.; Flores, J. A.; Pellei, M.; Morresi, B.; Gioia Lobbia, G.; Singh, S.; Kobayashi, Y.; Yousufuddin, M.; Santini, C. Dalton Trans. 2011, 40, 8569-8580.

(28) Teichgräber, J.; Dechert, S.; Meyer, F. J. Organomet. Chem. 2005, 690, 5255-5263.

(29) Cronin, D. L.; Wilkinson, J. R.; Todd, L. J. J. Magn. Res. 1975, 17, 353-361.

(30) (a) Muetterties, E. L.; Alegranti, C. W. J. Am Chem. Soc. 1972, 94, 6386-6391. (b) Goel, R. G.; Pilon, P. Inorg. Chem. 1978, 17, 2876-2879.

(31) Santini, C.; Gioia Lobbia, G.; Pettinari, C.; Pellei, M.; Valle, G.; Calogero, S. Inorg. Chem. 1998, 37, 890-900.

(32) Orpen, A. G.; Brammer, L.; Allen, F. H.; Kennard, O.; Watson, D. G.; Taylor, R. J. Chem. Soc., Dalton Trans. 1989, S1-S83. 
(33) (a) Peringer, P.; Lusser, M. Inorg. Chem. 1985, 24, 109-110. (b) Peringer, P.; Lusser, M. Polyhedron 1987, 6, 655-657.

(34) (a) Pringle, P. G.; Shaw, B. L. J. Chem. Soc., Chem. Commun. 1982, 581-583. (b) Olmstead, M. M.; Lee, C.-L.; Balch, A. L. Inorg. Chem. 1982, 21, 2712-2716. (c) Dean, P. A. W.; Srivastava, R. S. Can. J. Chem. 1985, 63, 2829-2839. (d) Oberhauser, W.; Stampfl, T.; Haid, R.; Langes, C.; Bachmann, C.; Kopacka, H.; Ongania, K.-H.; Brüggeller, P. Polyhedron 2001, 20, 727-740.

(35) Reger, D. L.; Semeniuc, R. F.; Smith, M. D. Rev. Roum. Chim. 2002, 47, 1037-1046.

(36) Gioia Lobbia, G.; Hanna, J. V.; Pellei, M.; Pettinari, C.; Santini, C.; Skelton, B. W.; White, A. H. Dalton Trans. 2004, 951-958.

(37) (a) van der Ploeg, A. F. M. J.; van Koten, G. Inorg. Chim. Acta 1981, 51, 225-239. (b) Dean, P. A. W.; Vittal, J. J.; Srivastava, R. S. Can. J. Chem. 1987, 65, 2628-2633. (c) Obendorf, D.; Probst, M.; Peringer, P.; Falk, H.; Müller, N. J. Chem. Soc., Dalton Trans. 1988, 1709-1711. (d) Yuan, Z.; Dryden, N. H.; Vittal, J. J.; Puddephatt, R. J. Can. J. Chem. 1994, 72, 1605-1609. (e) Pettinari, C.; Ngoune, J.; Marinelli, A.; Skelton, B. W.; White, A. H. Inorg. Chim. Acta 2009, 362, 3225-3230.

(38) (a) Manikandan, P.; Subramoni, M.; Varghese, B.; Manoharan, P. T. J. Chem. Soc., Dalton Trans. 1998, 3219-3225. (b) Tsubomura, T.; Takahashi, N.; Saito, K.; Tsukuda, T. Chem. Lett. 2004, 33, 678-679. 
Table 1. Experimental data for the X-ray diffraction studies on 4, 5, 10, 14, 15, and 18.

\begin{tabular}{|c|c|c|c|c|c|c|}
\hline & $\mathbf{4 \cdot} 0.5 \mathrm{C}_{7} \mathrm{H}_{8}$ & $\mathbf{5 \cdot} \cdot 0.5 \mathrm{C}_{7} \mathrm{H}_{8}$ & $\mathbf{1 0} \cdot \mathrm{C}_{6} \mathrm{H}_{5} \mathrm{~F}$ & 14. $\mathrm{C}_{6} \mathrm{H}_{14}$ & 15 & 18. $2 \mathrm{CH}_{2} \mathrm{Cl}_{2}$ \\
\hline Formula & $\mathrm{C}_{6}{ }_{9} \mathrm{H}_{110} \mathrm{Cu}_{2} \mathrm{~F}_{6} \mathrm{~N}_{10} \mathrm{O}_{6} \mathrm{~S}_{2} \mathrm{Ti}_{6}$ & $\mathrm{C}_{69} \mathrm{H}_{110} \mathrm{Ag}_{2} \mathrm{~F}_{6} \mathrm{~N}_{10} \mathrm{O}_{6} \mathrm{~S}_{2} \mathrm{Ti}_{6}$ & $\mathrm{C}_{42} \mathrm{H}_{62} \mathrm{CuF}_{4} \mathrm{~N}_{5} \mathrm{O}_{3} \mathrm{STi}_{3}$ & $\mathrm{C}_{62} \mathrm{H}_{84} \mathrm{CuF}_{3} \mathrm{~N}_{4} \mathrm{O}_{3} \mathrm{P}_{2} \mathrm{STi}_{3}$ & $\mathrm{C}_{56} \mathrm{H}_{70} \mathrm{AgF}_{3} \mathrm{~N}_{4} \mathrm{O}_{3} \mathrm{P}_{2} \mathrm{STi}_{3}$ & $\mathrm{C}_{90} \mathrm{H}_{124} \mathrm{Cl}_{4} \mathrm{Cu}_{2} \mathrm{~F}_{6} \mathrm{~N}_{8} \mathrm{O}_{6} \mathrm{P}_{2} \mathrm{~S}_{2} \mathrm{Ti}_{6}$ \\
\hline$M_{\mathrm{r}}$ & 1768.27 & 1856.93 & 1000.27 & 1291.57 & 1249.73 & 2210.31 \\
\hline$T[\mathrm{~K}]$ & $200(2) \mathrm{K}$ & 200(2) K & $100(2)$ & $200(2)$ & $200(2)$ & $200(2)$ \\
\hline$\lambda[\AA]$ & 0.71073 & 0.71073 & 0.71073 & 0.71073 & 0.71073 & 0.71073 \\
\hline crystal system & Monoclinic & Monoclinic & Monoclinic & Triclinic & Monoclinic & Triclinic \\
\hline space group & $C 2 / c$ & $C 2 / c$ & $P 2{ }_{1} / c$ & $P-1$ & $P 2_{1} / c$ & P-1 \\
\hline$a[\AA]$ & $35.874(7)$ & $36.798(6)$ & $11.236(1)$ & $11.416(2) ; 88.82(1)$ & $19.617(2)$ & $12.530(3) ; 89.02(1)$ \\
\hline$b[\AA] ; \beta\left[^{\circ}\right]$ & $12.173(4) ; 120.40(1)$ & $12.156(2) ; 121.82(1)$ & 17.283(4); 94.77(1) & $14.482(3) ; 88.81(1)$ & 14.188(3); 98.49(1) & $12.621(3) ; 71.72(1)$ \\
\hline$c[\AA] ; \gamma\left[{ }^{\circ}\right]$ & 21.981(3) & $22.225(3)$ & $25.424(6)$ & $19.792(3) ; 77.81(2)$ & $21.844(2)$ & 18.703(2); 68.81(1) \\
\hline$V\left[\AA^{3}\right]$ & $8279(4)$ & $8447(2)$ & 4920(2) & 3198(1) & 6013(2) & $2602.9(8)$ \\
\hline $\mathrm{Z}$ & 4 & 4 & 4 & 2 & 4 & 1 \\
\hline$\rho_{\text {calcd }}\left[\mathrm{g} \mathrm{cm}^{-3}\right]$ & 1.419 & 1.460 & 1.350 & 1.341 & 1.381 & 1.410 \\
\hline$\mu_{\mathrm{MoK \alpha}}\left[\mathrm{mm}^{-1}\right]$ & 1.167 & 1.104 & 0.993 & 0.826 & 0.846 & 1.071 \\
\hline$F(000)$ & 3672 & 3816 & 2080 & 1352 & 2576 & 1142 \\
\hline $\begin{array}{l}\text { crystal size } \\
{\left[\mathrm{mm}^{3}\right]}\end{array}$ & $0.30 \times 0.20 \times 0.10$ & $0.37 \times 0.29 \times 0.26$ & $0.24 \times 0.18 \times 0.18$ & $0.20 \times 0.14 \times 0.11$ & $0.17 \times 0.14 \times 0.11$ & $0.26 \times 0.13 \times 0.12$ \\
\hline$\theta$ range $(\mathrm{deg})$ & 3.04 to 27.50 & 5.05 to 27.51 & 3.04 to 27.51 & 3.04 to 27.50 & 3.02 to 27.50 & 3.04 to 27.50 \\
\hline index ranges & $\begin{array}{l}-46 \text { to } 46 \\
-15 \text { to } 15 \\
-28 \text { to } 27\end{array}$ & $\begin{array}{l}-47 \text { to } 47 \\
-15 \text { to } 15 \\
-28 \text { to } 28\end{array}$ & $\begin{array}{l}-14 \text { to } 14, \\
-22 \text { to } 22, \\
0 \text { to } 32\end{array}$ & $\begin{array}{l}-14 \text { to } 14, \\
-18 \text { to } 18, \\
-25 \text { to } 25\end{array}$ & $\begin{array}{l}-25 \text { to } 25, \\
-18 \text { to } 18, \\
0 \text { to } 28\end{array}$ & $\begin{array}{l}-16 \text { to } 16 \\
-16 \text { to } 16 \\
-24 \text { to } 23\end{array}$ \\
\hline $\begin{array}{l}\text { Reflections } \\
\text { collected }\end{array}$ & 91441 & 92545 & 93139 & 116550 & 138673 & 57759 \\
\hline Unique data & $9512[\mathrm{R}$ (int) $=0.073]$ & $9637[\mathrm{R}(\mathrm{int})=0.164]$ & $11283[\mathrm{R}(\mathrm{int})=0.052]$ & $14673[\mathrm{R}(\mathrm{int})=0.102]$ & $13812[\mathrm{R}$ (int) $=0.068]$ & $11946[\mathrm{R}(\mathrm{int})=0.104]$ \\
\hline $\begin{array}{l}\text { obsd data } \\
{[I>2 \sigma(I)]}\end{array}$ & 7160 & 5604 & 7727 & 10232 & 8420 & 7170 \\
\hline $\begin{array}{l}\text { Goodness-of-fit } \\
\text { on } \mathrm{F}^{2}\end{array}$ & 1.089 & 1.023 & 1.097 & 1.111 & 1.043 & 1.031 \\
\hline $\begin{array}{l}\text { final } R^{a} \text { indices } \\
{[I>2 \sigma(I)]}\end{array}$ & $\begin{array}{l}\mathrm{R} 1=0.040, \\
\mathrm{wR} 2=0.102\end{array}$ & $\begin{array}{l}\mathrm{R} 1=0.055 \\
\mathrm{wR} 2=0.105\end{array}$ & $\begin{array}{l}\mathrm{R} 1=0.061, \\
\mathrm{wR} 2=0.164\end{array}$ & $\begin{array}{l}\mathrm{R} 1=0.073, \\
\mathrm{wR} 2=0.220\end{array}$ & $\begin{array}{l}\mathrm{R} 1=0.051, \\
\mathrm{wR} 2=0.123\end{array}$ & $\begin{array}{l}\mathrm{R} 1=0.058 \\
\mathrm{wR} 2=0.117\end{array}$ \\
\hline $\begin{array}{l}R^{a} \text { indices (all } \\
\text { data) }\end{array}$ & $\begin{array}{l}\mathrm{R} 1=0.064, \\
\mathrm{wR} 2=0.111\end{array}$ & $\begin{array}{l}\mathrm{R} 1=0.128, \\
\mathrm{wR} 2=0.120\end{array}$ & $\begin{array}{l}\text { R1 = 0.094; } \\
\text { wR2 }=0.178\end{array}$ & $\begin{array}{l}\mathrm{R} 1=0.106, \\
\mathrm{wR} 2=0.245\end{array}$ & $\begin{array}{l}\mathrm{R} 1=0.104, \\
\mathrm{wR} 2=0.144\end{array}$ & $\begin{array}{l}\mathrm{R} 1=0.125, \\
\mathrm{wR} 2=0.135\end{array}$ \\
\hline $\begin{array}{l}\text { largest diff. } \\
\text { peak/hole }\left[\mathrm{e} . \AA^{-3}\right]\end{array}$ & 0.514 and -0.738 & 0.624 and -0.664 & 1.045 and -0.693 & 1.230 and -1.167 & 0.713 and -0.585 & 0.669 and -0.657 \\
\hline
\end{tabular}

${ }^{a} R 1=\Sigma|| \mathrm{F}_{0}|-| \mathrm{F}_{\mathrm{c}} \| /\left[\Sigma\left|\mathrm{F}_{0}\right|\right] \quad w R 2=\left\{\left[\Sigma w\left(F_{0}^{2}-F_{c}^{2}\right)^{2}\right] /\left[\Sigma w\left(F_{0}^{2}\right)^{2}\right]\right\}^{1 / 2}$ 